\title{
Chemical Analysis of Dietary Constituents in Rosa roxburghii and Rosa sterilis Fruits
}

\author{
Meng-Hua Liu ${ }^{1}$, Qi Zhang ${ }^{1}$, Yuan-He Zhang ${ }^{2}$, Xian-Yuan Lu ${ }^{1}$, Wei-Ming Fu ${ }^{1,2, *}$ and \\ Jing-Yu He ${ }^{2, *}$ \\ 1 Guangdong Provincial Key Laboratory of New Drug Screening, School of Pharmaceutical Sciences, \\ Southern Medical University, Guangzhou 510515, Guangdong, China; \\ menghua_liu@hotmail.com (M.-H.L.); qizhang100@sina.com (Q.Z.); lxy211723@126.com (X.-Y.L.) \\ 2 Bioengineering Research Centre, Guangzhou Institute of Advanced Technology, \\ Chinese Academy of Sciences, Guangzhou 511458, Guangdong, China; zhyuanhe@mail2.sysu.edu.cn \\ * Correspondence: fuweiming76@smu.edu.com (W.-M.F.); jy.he@giat.ac.cn (J.-Y.H.); \\ Tel.: +86-20-2291-2742 (J.-Y.H.); Fax: +86-20-2291-2525 (J.-Y.H.)
}

Academic Editor: Derek J. McPhee

Received: 21 June 2016; Accepted: 3 September 2016; Published: 9 September 2016

\begin{abstract}
Both Rosa roxburghii and R. sterilis, belonging to the Rosaceae, are endemic species in Guizhou Province, China. The fruits of these two species are mixed-used as functional food in the region. Aiming to elucidate the phytochemical characteristics of $R$. roxburghii and $R$. sterilis fruits, the essential oils and constituents in a methanol extract have been analyzed and compared by GC-MS and UFLC/Q-TOF-MS, respectively. As a result, a total of 135 volatile compounds were identified by GC-MS and 91 components were different between $R$. roxburghii and $R$. sterilis fruits; a total of 59 compounds in methanol extracts were identified by UFLC/Q-TOF-MS, including 13 organic acids, 12 flavonoids, 11 triterpenes, nine amino acids, five phenylpropanoid derivatives, four condensed tannins, two stilbenes, two benzaldehyde derivatives and one benzoic acid derivative; and nine characteristic compounds were found between $R$. roxburghii and $R$. sterilis fruits. This systematic study plays an important role for $R$. roxburghii and $R$. sterilis fruits in the product development.
\end{abstract}

Keywords: Rosa roxburghii; Rosa sterilis; constituents; GC-MS; UFLC/Q-TOF-MS

\section{Introduction}

Rosa roxburghii (Figure 1), belonging to the Rosaceae family, originates from the karst areas of Guizhou Province, China and the effect of promoting digestion of its fruit was firstly recorded in "Ben-cao-gang-mu-shi-yi" in 1765 A.D. [1]. Modern pharmacological studies have proven that R. roxburghii fruit processes antioxidant, antimutagenic, antiatherogenic and antitumor effects, as well as genoprotective and radioprotective activities [2-5]. Due to the beneficial effects, a number of phytochemical studies have been performed on this species, and various phytochemicals, including flavonoids, organic acids, triterpenes, amino acids and essential oils have been found in $R$. roxburghii fruit [3,6-10]. Among them, the flavonoids and organic acids in $R$. roxburghii fruit have been widely studied. The total flavonoid content in R. roxburghii fruit was 5981-12,895 mg/100 g dry weight, which was approximately 120-360 folds that in citrus [11]. The total content of the six organic acids (malic acid, lactic acid, tartaric acid, citric acid, oxalic acid and succinic acid) in $R$. roxburghii fruit was over $40 \mathrm{mg} / \mathrm{g}$ fresh weight [5,12]. Moreover, ascorbic acid, a well-known organic acid, was the most prevalent compound with 4500-6800 mg/100 g dry weight in $R$. roxburghii fruit [13]. To our best knowledge, ascorbic acid is an essential nutrient related to the biosynthesis of collagen and certain hormones, and is a potential substance to reduce the risk of some diseases (e.g., cancer, cardiovascular and neurodegenerative diseases). In view of the high contents and bioactive properties of flavonoids and 
ascorbic acid, $R$. roxburghii fruit has increasing applications to produce juice, wine and the preserved fruit can be used as a dietary supplement in the health-related industries.

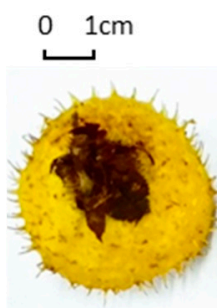

A

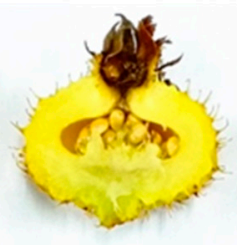

B

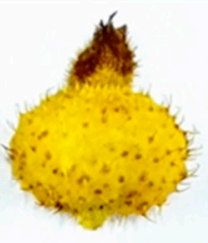

C

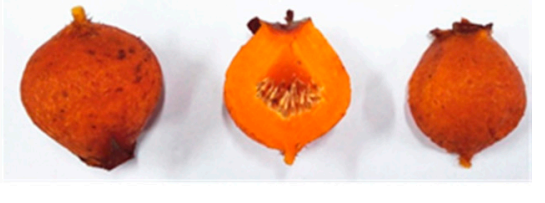

$\mathrm{D}$
E
F

Figure 1. Morphology of $R$. roxburghii (A-C) and R. sterilis (D-F) fruits.

R. sterilis (Rosaceae, Figure 1) is a newly found species in Anshun, Guizhou Province, described by Shengde Shi in 1985 [14]. R. sterilis have a very close genetic relationship to $R$. roxburghii based on Random Amplified Polymorphic DNA (RAPD) markers [15]. Recently, R. sterilis fruit has been mixed with $R$. roxburghii fruit in the food industry due to the fact they come from the same producing region (mainly in Guizhou Province) and similar taste. It is well known that the constituents that are responsible for the flavor and the bioactivities have a great effect on the application of plant materials. Up to now, a few kinds of ingredients in R. sterilis fruit, namely essential oils, triterpenes, amino acids and trace elements have been identified [14,16-18]. However, it is still difficult to estimate whether $R$. sterilis fruit could be used as a substitute for $R$. roxburghii fruit in the food industry. Thus, it is necessary to elucidate the chemical profiles of $R$. roxburghii and $R$. Sterilis fruits before these two fruits are well developed.

In recent years, gas chromatography-mass spectrometer (GC-MS) and ultra-fast liquid chromatography/quadrupole time-of-flight mass spectrometry (UFLC/Q-TOF-MS) have become powerful technologies for chemical identification in complex extracts due to their high resolution and low detection limit [19-22]. Therefore, in this study, the essential oils and constituents in methanol extracts of $R$. roxburghii and $R$. sterilis fruits were identified and compared by GC-MS and UFLC/Q-TOF-MS to elucidate their chemical characteristics.

\section{Results and Discussion}

\subsection{Chemical Analysis and Comparison of Essential Oils by GC-MS}

It is well known that essential oils comprise an important part of fruits. In this study, a total of 135 compounds were identified in $R$. roxburghii and R. sterilis fruits by GC-MS. As shown in Table 1 and Figure 2, R. roxburghii and $R$. sterilis fruits differed in the composition of their essential oils, and only 45 components were shared between them. Interestingly, aliphatic compounds were the major constituents in essential oils of $R$. roxburghii and $R$. sterilis fruits. In $R$. roxburghii fruit, 89 compounds were found, representing $98.88 \%$ of the essential oil and the ten components with higher relative peak area were $n$-hexadecanoic acid (16.06\%), octadecane (8.16\%), 9,12,15-octadecatrien-1-ol (6.66\%), nonacosane $(6.44 \%)$, cholestane $(5.24 \%), \beta$-sitosterol $(4.60 \%)$, stigmastane $(4.44 \%)$, tetracosane $(3.24 \%)$, 9,12-octadecadienoic acid (2.92\%), and 2,2'-methylenebis[6-(1,1-dimethylethyl)-4-methyl]phenol $(2.78 \%) ; 91$ compounds represented $93.19 \%$ of the essential oil of $R$. sterilis fruit and the ten components with higher relative peak area were $n$-hexadecanoic acid (20.86\%), 9,12,15-octadecatrienoic acid $(9.06 \%)$, octadecane $(5.70 \%)$, heptadecane $(5.38 \%)$, nonacosane $(5.07 \%)$, cholestane $(3.93 \%)$, stigmastane $(3.27 \%)$, triacontane (2.88\%), 2,2'-methylenebis[6-(1,1-dimethylethyl)-4-methyl]phenol (2.51\%) and $\beta$-sitosterol $(2.36 \%)$. The essential oils of $R$. roxburghii and $R$. sterilis fruits have been reported previously $[8,11,12,14-16]$. The essential oil compositions varied highly when different extraction methods were used, such as hydrodistillation, supercritical $\mathrm{CO}_{2}$ extraction, solvent extraction and 
solid-phase microextraction. Liang et al. [23] compared the volatile compounds of $R$. roxburghii fruit extracted by hydrodistillation and solvent extraction, and found that hydrodistillation was the most effective approach for the extraction of long chain fatty acids, such as hexadecanoic acid and 9,12-octadecadienoic acid, which is accord with our results. Zhang et al. [16] separated and identified 41 volatile compounds from $R$. roxburghii fruit by solid-phase microextraction and GC-MS, and confirmed that limonene, ethyl caprylate, ethyl caproate, $\beta$-chamigrene and guaiene were the main constituents. A total of 57 volatile compounds from $R$. sterilis fruit have been reported by Jiang et al. [24] and 1,2,3,4-tetrahydro-1,1,6-trimethylnaphthalene, tetradecane, $\beta$-selinene, hexanoic acid and dihydro- $\beta$-ionol were the main constituents, which were obviously different from those ( $\beta$-sitosterol, hentriacontane, octacosane, hexanoic acid and 11-(pentan-3-yl) henicosane) obtained by supercritical $\mathrm{CO}_{2}$ extraction [25]. Moreover, a higher relative intensity was found in the GC-MS chromatogram of $R$. sterilis fruit, indicating the content of essential oils in $R$. sterilis fruit was more than in $R$. roxburghii fruit using the same extraction method. This was consistent with the reports that extraction rates of essential oils were $1.8 \%$ and 0.8 for $R$. sterilis and R. roxburghii, respectively $[23,25]$. Among the 45 shared compounds, most of them have higher relative intensity and peak area in $R$. sterilis fruit than in $R$. roxburghii fruit, except for 5,6-dimethyldecane (17). In the present study, more constituents were separated and identified in the essential oils of $R$. roxburghii and $R$. sterilis fruits than in the previous studies using hydrodistillation. Ninety-one volatile compounds were different in these two species, which might explain their different smell and flavor.

Some main constituents in the essential oils have been reported for their pharmacological activities and nutritional values. For example, stigmastane and its derivatives possess anti-herpes virus and anti-inflammatory effects [26]. Meanwhile, 9,12,15-octadecatrienoic acid (linolenic acid), known as a vascular scavenger, has preventative effects against cardiovascular diseases, such as softening heart and brain blood vessels, promoting blood circulation and lowering blood pressure [27-29]. Therefore, elucidating the composition of the essential oils of R. roxburghii and R. sterilis fruits is useful for product development.
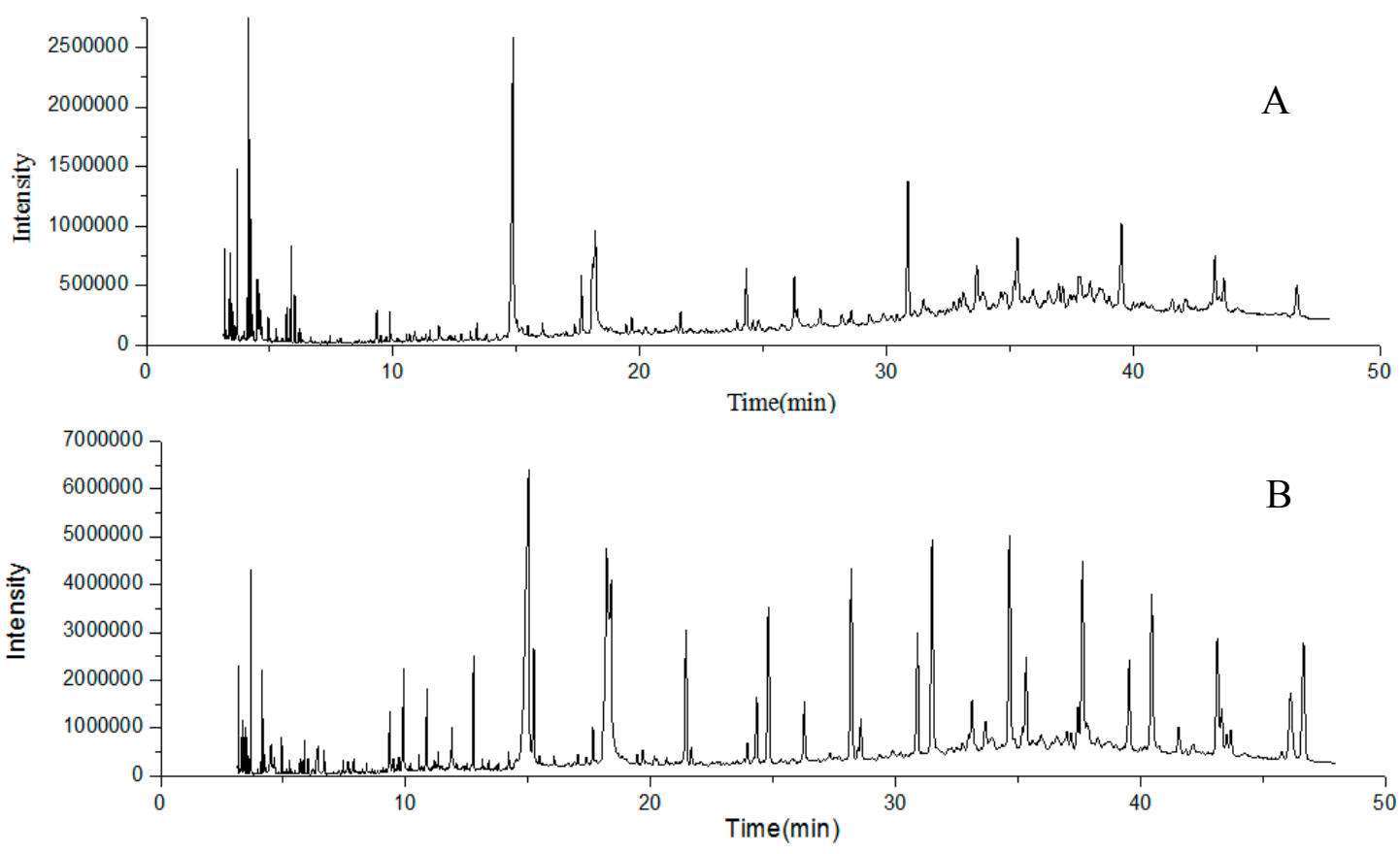

Figure 2. The representative total ion chromatograms of R. roxburghii (A) and R. sterilis (B) fruits obtained from GC-MS analysis. 
Table 1. Volatile compounds identified in R. roxburghii and R. sterilis fruits by GC-MS $(n=3)$.

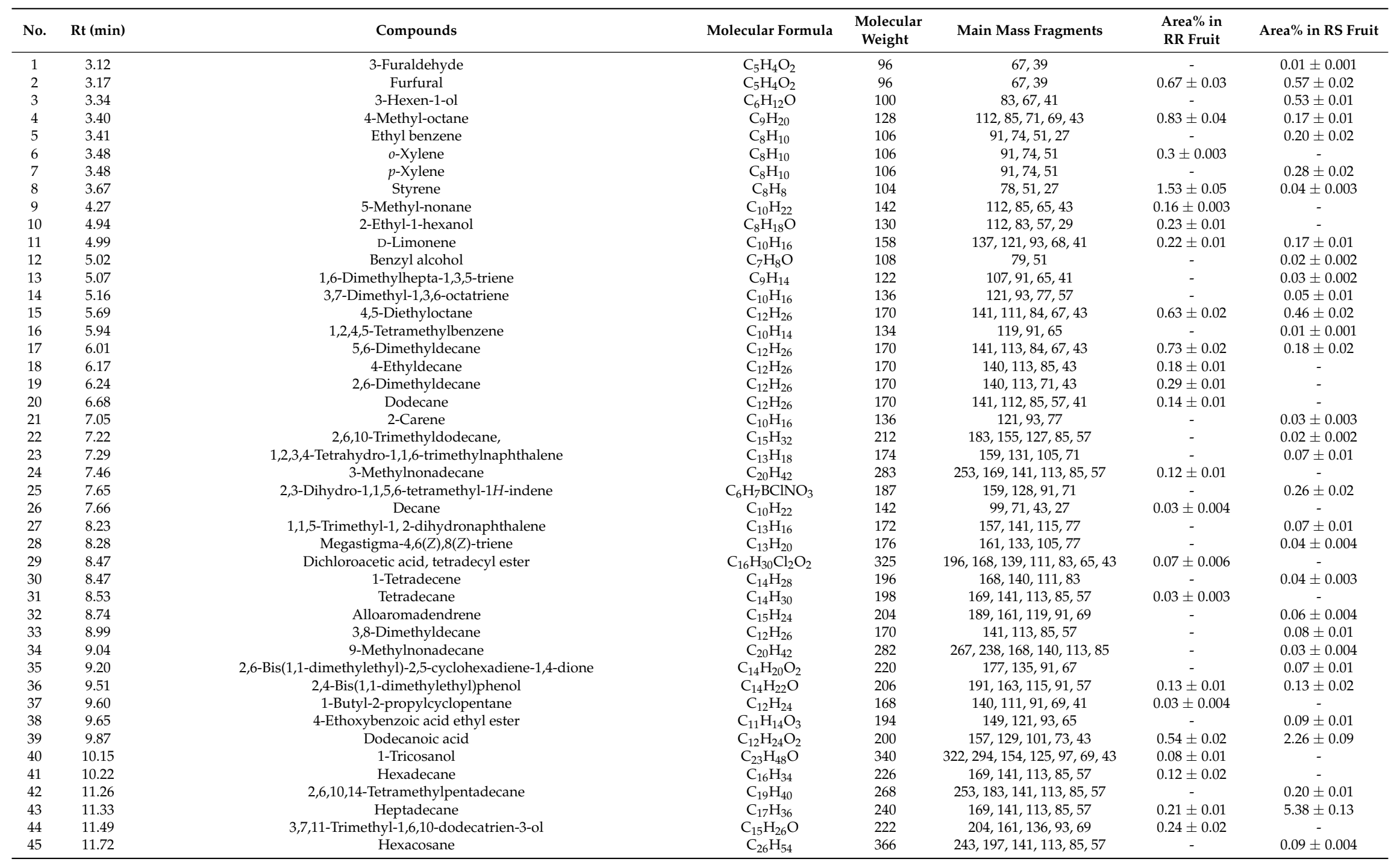


Table 1. Cont

\begin{tabular}{|c|c|c|c|c|c|c|c|}
\hline No. & Rt (min) & Compounds & Molecular Formula & $\begin{array}{l}\text { Molecular } \\
\text { Weight }\end{array}$ & Main Mass Fragments & $\begin{array}{l}\text { Area\% in } \\
\text { RR Fruit }\end{array}$ & Area $\%$ in RS Fruit \\
\hline 46 & 11.83 & 9-Octylheptadecane & $\mathrm{C}_{25} \mathrm{H}_{52}$ & 352 & $239,197,169,141,113,85,57$ & $0.09 \pm 0.01$ & - \\
\hline 47 & 11.86 & Tetradecanoic acid & $\mathrm{C}_{14} \mathrm{H}_{28} \mathrm{O}_{2}$ & 228 & $185,157,129,97,73$ & $0.44 \pm 0.02$ & $0.92 \pm 0.02$ \\
\hline 48 & 12.08 & Benzyl benzoate & $\mathrm{C}_{14} \mathrm{H}_{12} \mathrm{O}_{2}$ & 212 & $167,105,77,51$ & - & $0.27 \pm 0.01$ \\
\hline 49 & 12.23 & 6-Tetradecanesulfonic acid butyl ester & $\mathrm{C}_{18} \mathrm{H}_{38} \mathrm{O}_{3} \mathrm{~S}$ & 334 & $196,127,91,71,52$ & - & $0.08 \pm 0.01$ \\
\hline 50 & 12.28 & 3,3,4-Trimethyl-4-p-tolylcyclopentanol & $\mathrm{C}_{15} \mathrm{H}_{22} \mathrm{O}$ & 218 & $200,163,147,119,91$ & $0.20 \pm 0.01$ & - \\
\hline 51 & 12.50 & 2,6,10,14-Tetramethylhexadecane & $\mathrm{C}_{20} \mathrm{H}_{42}$ & 282 & $253,183,141,113,85$ & $0.18 \pm 0.01$ & - \\
\hline 52 & 13.06 & 2-Methylheptadecane & $\mathrm{C}_{18} \mathrm{H}_{38}$ & 254 & $239,211,141,113,85,57$ & - & $0.11 \pm 0.01$ \\
\hline 53 & 13.25 & Cyclohexadecane & $\mathrm{C}_{16} \mathrm{H}_{32}$ & 224 & 125,55 & - & $0.16 \pm 0.02$ \\
\hline 54 & 13.40 & 1,2-Benzenedicarboxylic acid bis(2-methylpropyl) ester & $\mathrm{C}_{16} \mathrm{H}_{22} \mathrm{O}_{4}$ & 278 & $223,167,149,104,76,57$ & $0.44 \pm 0.03$ & $0.28 \pm 0.01$ \\
\hline 55 & 13.49 & Cyclopentadecane & $\mathrm{C}_{15} \mathrm{H}_{30}$ & 210 & $182,139,111,83,55$ & $0.07 \pm 0.001$ & - \\
\hline 57 & 13.68 & Hentriacontane & $\mathrm{C}_{31} \mathrm{H}_{64}$ & 436 & $169,141,113,85,57$ & & $0.14 \pm 0.01$ \\
\hline 58 & 13.69 & 8-Heptadecene & $\mathrm{C}_{17} \mathrm{H}_{34}$ & 238 & $210,140,111,83,55$ & $0.08 \pm 0.001$ & - \\
\hline 59 & 14.21 & 2,3-Dimethylnonadecane & $\mathrm{C}_{21} \mathrm{H}_{44}$ & 297 & $253,183,155,127,99,71$ & $0.19 \pm 0.01$ & - \\
\hline 60 & 14.53 & Palmitoleic acid & $\mathrm{C}_{16} \mathrm{H}_{30} \mathrm{O}_{2}$ & 254 & $236,192,137,111,83$ & $0.77 \pm 0.02$ & - \\
\hline 61 & 14.57 & Oxacycloheptadecan-2-one & $\mathrm{C}_{16} \mathrm{H}_{30} \mathrm{O}_{2}$ & 254 & $236,194,138,111,83$ & $0.39 \pm 0.01$ & - \\
\hline 62 & 14.87 & $n$-Hexadecanoic acid ${ }^{\text {a }}$ & $\mathrm{C}_{16} \mathrm{H}_{32} \mathrm{O}_{2}$ & 256 & $213,185,157,129,83,60$ & $16.06 \pm 0.25$ & $20.86 \pm 0.31$ \\
\hline 63 & 15.64 & 1-Heptacosanol & $\mathrm{C}_{27} \mathrm{H}_{56} \mathrm{O}$ & 396 & $378,181,153,125,97,57$ & $0.29 \pm 0.02$ & - \\
\hline 64 & 16.06 & 3,7,11,15-Tetramethylhexadeca-1,3,6,10,14-pentaene & $\mathrm{C}_{20} \mathrm{H}_{32}$ & 272 & $229,191,119,93,69$ & $0.65 \pm 0.01$ & - \\
\hline 65 & 16.07 & 3,7,11-Trimethyl-2,6,10-dodecatrien-1-ol & $\mathrm{C}_{15} \mathrm{H}_{26} \mathrm{O}$ & 222 & $191,161,137,93,69$ & - & $0.70 \pm 0.01$ \\
\hline 66 & 16.12 & Trispiro[4.2.4.2.4.2.]heneicosane & $\mathrm{C}_{21} \mathrm{H}_{44}$ & 296 & $288,231,192,163,135,97$ & $0.29 \pm 0.01$ & - \\
\hline 67 & 16.47 & N-[4-bromo-n-butyl]-2-piperidinone & $\mathrm{C}_{9} \mathrm{H}_{16} \mathrm{BrNO}$ & 234 & $205,154,97,43$ & - & $0.10 \pm 0.01$ \\
\hline 68 & 16.73 & 2,2-Dimethyl-5-(3-methyloxiranyl)-cyclohexanone & $\mathrm{C}_{11} \mathrm{H}_{20} \mathrm{O}_{2}$ & 196 & $182,153,123,95,69,41$ & $0.11 \pm 0.008$ & - \\
\hline 71 & 17.04 & 8-Hexadecene & $\mathrm{C}_{16} \mathrm{H}_{32}$ & 224 & $196,153,125,97,69$ & - & $0.60 \pm 0.02$ \\
\hline 72 & 17.21 & Estra-1,3,5(10)-trien-17 $\beta$-ol & $\mathrm{C}_{18} \mathrm{H}_{24} \mathrm{O}$ & 256 & $185,157,129,97,73$ & - & $0.11 \pm 0.01$ \\
\hline 73 & 17.23 & Nonadecyl pentafluoropropionate & $\mathrm{C}_{22} \mathrm{H}_{39} \mathrm{~F}_{5} \mathrm{O}_{2}$ & 430 & $313,266,153,125,97,57$ & $0.03 \pm 0.001$ & - \\
\hline 74 & 17.36 & Heneicosane & $\mathrm{C}_{21} \mathrm{H}_{44}$ & 296 & $197,169,141,113,85$ & $0.63 \pm 0.02$ & $0.51 \pm 0.02$ \\
\hline 75 & 17.64 & 6-Octen-1-ol, 3,7-dimethyl acetate & $\mathrm{C}_{12} \mathrm{H}_{22} \mathrm{O}_{2}$ & 198 & $156,123,103,81$ & $2.33 \pm 0.15$ & - \\
\hline 76 & 17.94 & Trifluoroacetic acid pentadecyl ester & $\mathrm{C}_{18} \mathrm{H}_{31} \mathrm{~F}_{3} \mathrm{O}_{2}$ & 336 & $306,255,182,140,111,83$ & & $0.42 \pm 0.02$ \\
\hline 77 & 18.09 & 9,12-Octadecadienoic acid a & $\mathrm{C}_{18} \mathrm{H}_{32} \mathrm{O}_{2}$ & 280 & $236,150,123,95,67$ & $2.92 \pm 0.09$ & $0.59 \pm 0.01$ \\
\hline 78 & 18.21 & 9,12,15-Octadecatrien-1-ol & $\mathrm{C}_{18} \mathrm{H}_{32} \mathrm{O}$ & 264 & $236,208,108,79$ & $6.66 \pm 0.14$ & - \\
\hline 79 & 18.40 & 9,12,15-Octadecatrienoic acid ${ }^{\text {a }}$ & $\mathrm{C}_{18} \mathrm{H}_{30} \mathrm{O}_{2}$ & 278 & $222,163,135,108,79$ & - & $9.06 \pm 0.17$ \\
\hline 80 & 18.66 & 4-(4-Ethylcyclohexyl)-1-pentylcyclohexene & $\mathrm{C}_{19} \mathrm{H}_{34}$ & 262 & $220,191,164,123,81$ & $0.42 \pm 0.01$ & - \\
\hline 81 & 18.66 & Methyl $6,9,12$-hexadecatrienoate & $\mathrm{C}_{17} \mathrm{H}_{28} \mathrm{O}_{2}$ & 264 & $194,175,135,107,79$ & - & $0.69 \pm 0.02$ \\
\hline 82 & 18.80 & 9-Octadecenoic acid ${ }^{\text {a }}$ & $\mathrm{C}_{18} \mathrm{H}_{34} \mathrm{O}_{2}$ & 282 & $264,222,165,137,111,83$ & $0.43 \pm 0.01$ & $0.14 \pm 0.01$ \\
\hline 83 & 18.83 & 1-Eicosene & $\mathrm{C}_{20} \mathrm{H}_{40}$ & 280 & $252,182,153,125,97$ & $0.53 \pm 0.02$ & $0.10 \pm 0.01$ \\
\hline 84 & 18.84 & tert-Hexadecanethiol & $\mathrm{C}_{16} \mathrm{H}_{34} \mathrm{~S}$ & 258 & $224,165,111,57$ & - & $0.74 \pm 0.01$ \\
\hline 85 & 18.97 & Bacchotricuneatin $C$ & $\mathrm{C}_{20} \mathrm{H}_{22} \mathrm{O}_{5}$ & 342 & $245,191,145,112,71$ & - & $0.14 \pm 0.01$ \\
\hline 86 & 19.01 & E-8-Methyl-7-dodecen-1-ol acetate & $\mathrm{C}_{15} \mathrm{H}_{28} \mathrm{O}_{2}$ & 240 & $197,165,126,97,69$ & $0.08 \pm 0.003$ & - \\
\hline 87 & 19.01 & 3-Methylheptadecane & $\mathrm{C}_{18} \mathrm{H}_{38}$ & 254 & $225,169,141,113,85,57$ & - & $0.12 \pm 0.01$ \\
\hline 88 & 19.03 & 1-Chloro-octadecane & $\mathrm{C}_{18} \mathrm{H}_{37} \mathrm{Cl}$ & 288 & $175,147,113,85,57$ & - & $0.50 \pm 0.01$ \\
\hline 89 & 19.15 & 2-Dodecen-1-yl(-)succinic anhydride & $\mathrm{C}_{16} \mathrm{H}_{26} \mathrm{O}_{3}$ & 266 & $237,299,181,149,123,97,69,41$ & $0.07 \pm 0.001$ & $0.10 \pm 0.01$ \\
\hline
\end{tabular}


Table 1. Cont.

\begin{tabular}{|c|c|c|c|c|c|c|c|}
\hline No. & Rt (min) & Compounds & Molecular Formula & $\begin{array}{l}\text { Molecular } \\
\text { Weight }\end{array}$ & Main Mass Fragments & $\begin{array}{l}\text { Area\% in } \\
\text { RR Fruit }\end{array}$ & Area $\%$ in RS Frui \\
\hline 90 & 19.21 & 1-(1,5-Dimethylhexyl)-4-(4-methylpentyl)cyclohexane & $\mathrm{C}_{20} \mathrm{H}_{40}$ & 280 & $191,166,123,97,69$ & $0.05 \pm 0.001$ & $0.27 \pm 0.01$ \\
\hline 91 & 19.68 & 9-Tricosene & $\mathrm{C}_{23} \mathrm{H}_{46}$ & 322 & $294,167,139,111,83$ & $0.95 \pm 0.03$ & $0.89 \pm 0.02$ \\
\hline 92 & 19.83 & 13-Tetradecen-1-ol acetate & $\mathrm{C}_{16} \mathrm{H}_{30} \mathrm{O}_{2}$ & 254 & $194,167,139,111,83$ & $0.13 \pm 0.01$ & - \\
\hline 93 & 20.55 & 1-Tricosene & $\mathrm{C}_{23} \mathrm{H}_{46}$ & 322 & $196,169,139,111,83,57$ & $0.05 \pm 0.001$ & - \\
\hline 94 & 20.92 & 1,7,11-Trimethyl-4-(1-methylethyl)cyclotetradecane & $\mathrm{C}_{20} \mathrm{H}_{40}$ & 280 & $236,204,165,125,97$ & $0.10 \pm 0.01$ & $0.12 \pm 0.01$ \\
\hline 95 & 21.03 & 1-Docosene & $\mathrm{C}_{23} \mathrm{H}_{46}$ & 308 & $223,181,139,97,57$ & $0.33 \pm 0.01$ & $0.18 \pm 0.01$ \\
\hline 96 & 21.34 & Eicosyl pentafluoropropionate & $\mathrm{C}_{23} \mathrm{H}_{41} \mathrm{~F}_{5} \mathrm{O}_{2}$ & 444 & $426,280,182,153,125,97$ & $0.09 \pm 0.005$ & $0.16 \pm 0.01$ \\
\hline 97 & 21.66 & Eicosane & $\mathrm{C}_{20} \mathrm{H}_{42}$ & 282 & $197,169,141,113,85$ & $1.10 \pm 0.04$ & $2.28 \pm 0.10$ \\
\hline 98 & 21.91 & Docosane & $\mathrm{C}_{22} \mathrm{H}_{46}$ & 310 & $197,169,141,113,85$ & $0.04 \pm 0.003$ & $0.37 \pm 0.01$ \\
\hline 99 & 22.05 & Nonadecane & $\mathrm{C}_{19} \mathrm{H}_{40}$ & 268 & $197,169,141,113,85$ & $0.68 \pm 0.01$ & - \\
\hline 101 & 22.61 & Behenyl chloride & $\mathrm{C}_{22} \mathrm{H}_{45} \mathrm{Cl}$ & 344 & $189,169,141,113,85$ & $0.44 \pm 0.01$ & $0.05 \pm 0.004$ \\
\hline 102 & 22.72 & 11,13-Dimethyl-12-tetradecen-1-ol acetate & $\mathrm{C}_{18} \mathrm{H}_{34} \mathrm{O}_{2}$ & 282 & $267,208,151,123,95,69$ & $0.10 \pm 0.02$ & $0.69 \pm 0.02$ \\
\hline 103 & 22.72 & 15-Isobutyl-(13 $\alpha \mathrm{H})$-isocopalane & $\mathrm{C}_{24} \mathrm{H}_{44}$ & 332 & $276,219,191,151,123,95$ & - & $0.41 \pm 0.01$ \\
\hline 104 & 24.21 & Cyclotetracosane & $\mathrm{C}_{24} \mathrm{H}_{48}$ & 336 & $308,167,139,111,83$ & $0.41 \pm 0.01$ & $0.49 \pm 0.01$ \\
\hline 105 & 24.32 & 2,2'-Methylenebis[6-(1,1-dimethylethyl)-4-methyl]phenol & $\mathrm{C}_{23} \mathrm{H}_{32} \mathrm{O}_{2}$ & 340 & $284,177,149,121,91$ & $2.78 \pm 0.09$ & $2.51 \pm 0.07$ \\
\hline 106 & 24.59 & Hexadecyloxirane & $\mathrm{C}_{18} \mathrm{H}_{36} \mathrm{O}$ & 268 & $250,211,166,138,111,82$ & $0.71 \pm 0.03$ & - \\
\hline 107 & 25.48 & Dotriacontyl pentafluoropropionate & $\mathrm{C}_{35} \mathrm{H}_{65} \mathrm{~F}_{5} \mathrm{O}_{2}$ & 612 & $594,448,181,139,97,57$ & $0.08 \pm 0.002$ & $0.13 \pm 0.01$ \\
\hline 108 & 26.38 & Tetratriacontane & $\mathrm{C}_{34} \mathrm{H}_{70}$ & 478 & $253,225,197,169,141,113,85$ & - & $0.39 \pm 0.02$ \\
\hline 109 & 26.66 & 12-Pentacosene & $\mathrm{C}_{25} \mathrm{H}_{50}$ & 350 & $350,181,153,125,97,69$ & $0.20 \pm 0.01$ & - \\
\hline 110 & 27.32 & Bis(2-ethylhexyl)phthalate & $\mathrm{C}_{24} \mathrm{H}_{38} \mathrm{O}_{4}$ & 390 & $279,180,149,104,57$ & $1.60 \pm 0.06$ & - \\
\hline 111 & 27.95 & Eicosyl trifluoroacetate & $\mathrm{C}_{22} \mathrm{H}_{41} \mathrm{~F}_{3} \mathrm{O}_{2}$ & 394 & $376,325,280,153,125,97$ & $0.06 \pm 0.002$ & - \\
\hline 112 & 28.50 & Heptacosyl trifluoroacetate & $\mathrm{C}_{29} \mathrm{H}_{55} \mathrm{~F}_{3} \mathrm{O}_{2}$ & 492 & $474,423,378,181,139,97$ & $0.09 \pm 0.01$ & - \\
\hline 114 & 29.31 & 2-Dodecen-1-yl(-)succinic anhydride & $\mathrm{C}_{16} \mathrm{H}_{26} \mathrm{O}_{3}$ & 266 & $209,166,137,97,69$ & $1.65 \pm 0.06$ & $0.08 \pm 0.002$ \\
\hline 115 & 29.53 & Tricosane & $\mathrm{C}_{23} \mathrm{H}_{48}$ & 324 & $197,169,141,113,85$ & $0.13 \pm 0.01$ & $0.53 \pm 0.02$ \\
\hline 116 & 29.88 & Pentacosane & $\mathrm{C}_{25} \mathrm{H}_{52}$ & 352 & $211,169,141,113,85,57$ & 年 & $1.56 \pm 0.05$ \\
\hline 117 & 29.89 & 2,6,10,14,18-Pentamethyleicosane & $\mathrm{C}_{25} \mathrm{H}_{52}$ & 352 & $253,183,141,113,85$ & $1.15 \pm 0.04$ & - \\
\hline 118 & 30.64 & 2,6,10,14-Tetramethyl-7-(3-methylpent-4-enylidene)pentadecane & $\mathrm{C}_{25} \mathrm{H}_{48}$ & 348 & $264,207,167,125,97$ & $0.56 \pm 0.02$ & - \\
\hline 119 & 30.67 & 14-Nonacosane & $\mathrm{C}_{29} \mathrm{H}_{60}$ & 408 & $378,181,153,125,97$ & $0.25 \pm 0.01$ & $0.10 \pm 0.03$ \\
\hline 120 & 30.87 & Octadecane & $\mathrm{C}_{18} \mathrm{H}_{38}$ & 254 & $169,141,113,85,57$ & $8.16 \pm 0.14$ & $5.70 \pm 0.09$ \\
\hline 121 & 31.05 & 1-Hexacosene & $\mathrm{C}_{26} \mathrm{H}_{52}$ & 364 & $209,181,153,125,97$ & $0.46 \pm 0.01$ & $0.54 \pm 0.02$ \\
\hline 122 & 31.59 & 1-Bromo-11-iodoundecane & $\mathrm{C}_{11} \mathrm{H}_{22} \mathrm{BrI}$ & 362 & $281,233,177,135,97$ & $0.31 \pm 0.01$ & $0.27 \pm 0.01$ \\
\hline 123 & 31.62 & 1-Methyl-4-(1-methylethyl)-3-[1-methyl-1-(4-methylpentyl)-5-methylheptyl]cyclohexene & $\mathrm{C}_{25} \mathrm{H}_{48}$ & 348 & $248,193,123,69$ & $0.24 \pm 0.01$ & - \\
\hline 124 & 32.46 & 13-Methyl-Z-14-nonacosene & $\mathrm{C}_{30} \mathrm{H}_{60}$ & 420 & $405,209,181,153,125,97$ & $0.78 \pm 0.02$ & \\
\hline 125 & 32.98 & $(5 \alpha, 14 \beta)$-Cholestane & $\mathrm{C}_{27} \mathrm{H}_{48}$ & 372 & $259,218,176,149,109$ & $2.11 \pm 0.03$ & $1.66 \pm 0.09$ \\
\hline 126 & 33.10 & Tetracosane & $\mathrm{C}_{24} \mathrm{H}_{50}$ & 338 & $169,141,113,85,57$ & $3.24 \pm 0.05$ & $1.00 \pm 0.07$ \\
\hline 127 & 33.67 & Cholestane & $\mathrm{C}_{27} \mathrm{H}_{48}$ & 372 & $262,217,149,109,81$ & $5.24 \pm 0.09$ & $3.93 \pm 0.10$ \\
\hline 128 & 34.33 & $(5 \alpha, 13 \alpha)$-D-Homoandrostane & $\mathrm{C}_{20} \mathrm{H}_{34}$ & 274 & $259,217,177,149,95,55$ & $1.25 \pm 0.04$ & $1.39 \pm 0.06$ \\
\hline 129 & 35.31 & Nonacosane & $\mathrm{C}_{29} \mathrm{H}_{60}$ & 408 & $197,169,141,113,85$ & $6.44 \pm 0.21$ & $5.07 \pm 0.19$ \\
\hline 130 & 35.94 & Stigmastane & $\mathrm{C}_{29} \mathrm{H}_{52}$ & 400 & $290,217,189,149,109$ & $4.44 \pm 0.18$ & $3.27 \pm 0.14$ \\
\hline 131 & 37.45 & 1-Iodo-octadecane & $\mathrm{C}_{18} \mathrm{H}_{37} \mathrm{I}$ & 380 & $253,183,141,99,57$ & $2.16 \pm 0.08$ & $0.67 \pm 0.03$ \\
\hline 132 & 37.46 & Triacontane & $\mathrm{C}_{30} \mathrm{H}_{62}$ & 422 & $197,169,141,113,85,57$ & - & $2.88 \pm 0.11$ \\
\hline 133 & 37.80 & 28-Nor-17 $\alpha(\mathrm{H})$-hopane & $\mathrm{C}_{29} \mathrm{H}_{50}$ & 398 & $383,355,218,191,137,109$ & $2.44 \pm 0.09$ & - \\
\hline 134 & 43.31 & $\beta$-Sitosterol & $\mathrm{C}_{29} \mathrm{H}_{50} \mathrm{O}$ & 414 & $381,329,255,213,145,81$ & $4.60 \pm 0.17$ & $2.36 \pm 0.09$ \\
\hline 135 & 43.70 & $(3 \beta, 24 Z)$-Stigmasta-5,24(28)-dien-3-ol & $\mathrm{C}_{29} \mathrm{H}_{48} \mathrm{O}$ & 412 & $379,314,281,229,202$ & - & $2.04 \pm 0.10$ \\
\hline
\end{tabular}

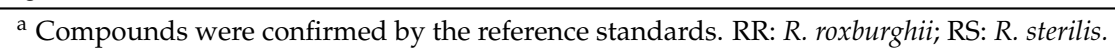




\subsection{Chemical Analysis and Comparison of Multiple Constituents by UFLC/Q-TOF-MS/MS}

\subsubsection{Identification of Constituents}

A total of 59 compounds were identified or tentatively characterized, including 13 organic acids (1, 5-6, 8-10, 12, 16, 19, 25, 28, 57 and 59), 12 flavonoids (18, 30-32, 34-36, 38, 44, 47, 48 and 49), 11 triterpenes $(43,45,46,50-56$ and 58), nine amino acids $(2-4,7,11,13,14,17$ and 20), five phenylpropanoid derivatives (26, 33, 39, 41 and 42), four condensed tannins (21, 24, 27 and 29), two stilbenes (37 and 40), two benzaldehyde derivatives (15 and 22) and one benzoic acid derivative (23). The detailed information is summarized in Table 2.

Organic acids: Compared with the reference standards, peaks 1, 5, 8, 9, 10, 12, 16, 19, 25, 28, 57 and 59 were identified directly as lactic acid, malic acid, ascorbic acid, protocatechuic acid, citric acid, $p$-coumaric acid, gallic acid, syringic acid, $p$-hydroxybenzoic acid, caffeic acid, 9,12,15-octadecatrienoic acid and 9,12-octadecadienoic acid, respectively. The characteristic fragments were $\left[\mathrm{M}-\mathrm{H}-\mathrm{H}_{2} \mathrm{O}\right]^{-}$, $\left[\mathrm{M}-\mathrm{H}-\mathrm{CO}_{2}\right]^{-}$and $[\mathrm{M}-\mathrm{H}-\mathrm{HCOOH}]^{-}$in negative ion mode. Compound 6 gave an $[\mathrm{M}-\mathrm{H}]^{-}$at $m / z$ 191.05529, corresponding to its elemental composition of $\mathrm{C}_{7} \mathrm{H}_{12} \mathrm{O}_{6}$. The high sensitive ions of $m / z$ 173.0462 resulted from the loss of $\mathrm{H}_{2} \mathrm{O}$, subsequently the loss of $\mathrm{HCOOH}$ yielded the ion $m / z$ 127.0396. Therefore, compound $\mathbf{6}$ was tentatively identified as quinic acid [30,31].

Flavonoids: Peaks 30, 31, 35, 44, 47 and 49 were identified as rutin, isoquercitrin, quercitrin, quercetin, kaempferol and luteolin, respectively. The characteristic fragment of flavonoid glycoside was at $m / z$ [aglycone] ${ }^{+}$in positive ion mode and [aglycone] ${ }^{-}$by the loss of glycoside in negative ion mode. Referring to the compounds reported in $R$. roxburghii, peaks 18 (molecular formula: $\mathrm{C}_{15} \mathrm{H}_{14} \mathrm{O}_{7}$ ), 32 (molecular formula: $\mathrm{C}_{27} \mathrm{H}_{28} \mathrm{O}_{16}$ ), 36 (molecular formula: $\mathrm{C}_{27} \mathrm{H}_{28} \mathrm{O}_{15}$ ) and 38 (molecular formula: $\mathrm{C}_{27} \mathrm{H}_{28} \mathrm{O}_{15}$ ) were tentatively identified as epigallocatechin [6], quercetin 3-O-[(6-O-3-hydroxy-3-methylglutaryl)- $\beta$-galactoside] [32], kaempferol 3-O-[(X-O-3-hydroxy-3-methylglutaryl)- $\beta$-galactoside $] \quad[32]$ and kaempferol 3-O-[(X-O-3-hydroxy-3-methylglutaryl)- $\beta$-glucoside] [32], respectively. Peak 34 showed a deprotonated molecule ion at $m / z 433.09253$ in negative ion mode. The determination of fragment ion at $\mathrm{m} / z 301$ confirmed the losses as $\mathrm{C}_{5} \mathrm{H}_{10} \mathrm{O}_{4}$, suggesting that the existence of xyloside group [9,33]. Peak 48 gave a protonated ion at $m / z 273.07551$ and the molecular formula was $\mathrm{C}_{15} \mathrm{H}_{12} \mathrm{O}_{5}$. According to MS data, fragment ion at $m / z 153$ was generated by retro Diels-Alder reaction in the $C$ ring, which is the same as that happened in the standard compounds of quercetin, kaempferol and luteolin. Therefore, it was tentatively identified as dihydroapigenin [33].

Triterpenes: Peak 58 was identified as ursolic acid by comparison with the reference standard. Peaks 43 and 45 were a pair of isomers with a $[\mathrm{M}-\mathrm{H}]^{-}$ion at $\mathrm{m} / \mathrm{z} 665$, corresponding to $\mathrm{C}_{36} \mathrm{H}_{58} \mathrm{O}_{11}$. The fragment ion at $m / z 485,180$ Da less than molecular weight, hinted the presence of a glucose group. Then $m / z 441$ appeared behind $m / z 485$ in peak 43 of MS spectrum, indicating the presence of a carboxyl group. Considering the retention time and different fragments, the glucose group was not linked to the carboxyl group directly. It was thus tentatively identified as polygalacic acid 3-O- $\beta$-D-glucopyranoside and peak 45 was tentatively identified as $19 \alpha$-hydroxyasiatic acid-28-O- $\beta$-D-glucopyranoside [34]. Peaks 46, 50 and 52 with $\mathrm{m} / z$ $649.39552\left(\mathrm{C}_{36} \mathrm{H}_{58} \mathrm{O}_{10}\right), 503.33973\left(\mathrm{C}_{30} \mathrm{H}_{48} \mathrm{O}_{6}\right)$ and $487.34276\left(\mathrm{C}_{30} \mathrm{H}_{48} \mathrm{O}_{5}\right)$ were tentatively identified as kajiichigoside F1, 1-hydroxyeuscaphic acid, and euscaphic acid, respectively, according to previous reports [7,10,14]. Peaks 51 and 53 had the same molecular formula of $\mathrm{C}_{30} \mathrm{H}_{46} \mathrm{O}_{5}$. Peak 53 lost neutral ions of $\mathrm{H}_{2} \mathrm{O}$ and $\mathrm{CO}_{2}$, then produced fragment ions of $m / z 467$ and 441 in MS spectrum, indicating the presence of hydroxyl and carboxyl groups. Therefore, peak 53 was identified as

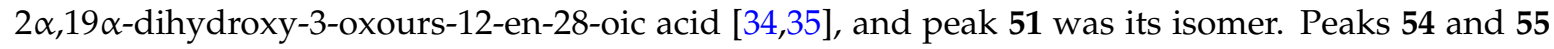
gave the same molecular formula and fragment ions in their MS spectra, and they were tentatively identified as pomolic acid or an isomer, which needs to be further confirmed by nuclear magnetic resonance (NMR) [7,10,14]. Peak 56 gave a $[\mathrm{M}-\mathrm{H}]^{-}$ion at $m / z 469.33187\left(\mathrm{C}_{30} \mathrm{H}_{46} \mathrm{O}_{4}\right)$. The fragment ions at $m / z 451$ and 407 were produced by the loss of $\mathrm{H}_{2} \mathrm{O}$ and the subsequent loss of $\mathrm{CO}_{2}$, and it was therefore tentatively identified as $2 \alpha, 3 \beta$-dihydroxylup-20(29)-en-28-oic acid [34]. 
Table 2. Compounds identified in the methanol extracts of $R$. roxburghii and R. sterilis fruits.

\begin{tabular}{|c|c|c|c|c|c|c|c|c|}
\hline No. & $\begin{array}{c}\mathrm{Rt} \\
(\mathrm{min})\end{array}$ & $\begin{array}{l}\text { Molecular } \\
\text { Formula }\end{array}$ & {$[\mathbf{M}+\mathbf{H}]^{+}$} & {$[\mathbf{M}-\mathbf{H}]^{-}$} & $\begin{array}{l}\text { Major Fragment Ions } \\
\text { in Positive Mode }\end{array}$ & $\begin{array}{l}\text { Major Fragment Ions } \\
\text { in Negative Mode }\end{array}$ & Identification & Source \\
\hline 1 & 3.28 & $\mathrm{C}_{3} \mathrm{H}_{6} \mathrm{O}_{3}$ & & $\begin{array}{c}89.02442 \\
(0)\end{array}$ & & $71.0233\left[\mathrm{M}-\mathrm{H}-\mathrm{H}_{2} \mathrm{O}\right]^{-}$ & Lactic acid $^{\text {a }}$ & RR, RS \\
\hline 2 & 3.36 & $\mathrm{C}_{3} \mathrm{H}_{7} \mathrm{NO}_{3}$ & $\begin{array}{c}106.04983 \\
(-0.4)\end{array}$ & $\begin{array}{c}104.0534 \\
(+0.1)\end{array}$ & $87.0324\left[\mathrm{M}+\mathrm{H}-\mathrm{NH}_{3}\right]^{+}$ & & Serine $^{\mathrm{a}}$ & RR, RS \\
\hline 3 & 3.4 & $\mathrm{C}_{6} \mathrm{H}_{14} \mathrm{~N}_{4} \mathrm{O}_{2}$ & $\begin{array}{c}175.11826 \\
(-4.0)\end{array}$ & $\begin{array}{c}173.10527 \\
(+5.0)\end{array}$ & $\begin{array}{l}158.0918\left[\mathrm{M}+\mathrm{H}-\mathrm{NH}_{3}\right]^{+}, \\
130.0970\left[\mathrm{M}+\mathrm{H}-\mathrm{NH}_{3}-\mathrm{CO}\right]^{+}, \\
116.0724\left[\mathrm{M}+\mathrm{H}-\mathrm{CH}_{5} \mathrm{~N}_{3}\right]^{+}\end{array}$ & & Arginine $^{a}$ & RR, RS \\
\hline 4 & 3.82 & $\mathrm{C}_{5} \mathrm{H}_{9} \mathrm{NO}_{2}$ & $\begin{array}{c}116.07018 \\
(-3.7)\end{array}$ & $\begin{array}{c}114.05579 \\
(-2.3)\end{array}$ & $70.0677[\mathrm{M}+\mathrm{H}-\mathrm{HCOOH}]^{+}$ & & Proline ${ }^{a}$ & RR, RS \\
\hline 5 & 3.92 & $\mathrm{C}_{4} \mathrm{H}_{6} \mathrm{O}_{5}$ & & $\begin{array}{c}133.01458 \\
(+2.5)\end{array}$ & & $115.0047\left[\mathrm{M}-\mathrm{H}-\mathrm{H}_{2} \mathrm{O}\right]^{-}$ & Malic acid ${ }^{a}$ & RR, RS \\
\hline 6 & 3.99 & $\mathrm{C}_{7} \mathrm{H}_{12} \mathrm{O}_{6}$ & $\begin{array}{l}193.06974 \\
(-4.8)\end{array}$ & $\begin{array}{l}191.05529 \\
(-4.3)\end{array}$ & & $\begin{array}{l}173.0462\left[\mathrm{M}-\mathrm{H}-\mathrm{H}_{2} \mathrm{O}\right]^{-} \\
127.0396\left[\mathrm{M}-\mathrm{H}-\mathrm{H}_{2} \mathrm{O}-\mathrm{HCOOH}\right]^{-} \\
109.0294\left[\mathrm{M}-\mathrm{H}-2 \mathrm{H}_{2} \mathrm{O}-\mathrm{HCOOH}\right]^{-}\end{array}$ & Quinic acid & RR, RS \\
\hline 7 & 4 & $\mathrm{C}_{5} \mathrm{H}_{11} \mathrm{NO}_{2}$ & $\begin{array}{c}118.08617 \\
(-0.7)\end{array}$ & $\begin{array}{c}116.07236 \\
(+5.7)\end{array}$ & $72.0828[\mathrm{M}+\mathrm{H}-\mathrm{HCOOH}]^{+}$ & & Valine $^{\mathrm{a}}$ & RR, RS \\
\hline 8 & 4.09 & $\mathrm{C}_{6} \mathrm{H}_{8} \mathrm{O}_{6}$ & $\begin{array}{c}177.03933 \\
(-0.2)\end{array}$ & $\begin{array}{c}175.02491 \\
(+0.6)\end{array}$ & $\begin{array}{l}\text { 129.0187, } \\
111.0080 \\
95.0138\end{array}$ & $\begin{array}{l}115.0043\left[\mathrm{M}-\mathrm{H}-\mathrm{C}_{2} \mathrm{H}_{4} \mathrm{O}_{2}\right]^{-} \\
87.0103\left[\mathrm{M}-\mathrm{H}-\mathrm{C}_{2} \mathrm{H}_{4} \mathrm{O}_{2}-\mathrm{CO}^{-}\right.\end{array}$ & Ascorbic acid ${ }^{a}$ & RR, RS \\
\hline 9 & 5.18 & $\mathrm{C}_{7} \mathrm{H}_{6} \mathrm{O}_{4}$ & $\begin{array}{c}155.03362 \\
(-1.7)\end{array}$ & $\begin{array}{c}153.01985 \\
(+3.4)\end{array}$ & $137.0253\left[\mathrm{M}+\mathrm{H}-\mathrm{H}_{2} \mathrm{O}\right]^{+}$ & 109.0.93 $\left[\mathrm{M}-\mathrm{H}-\mathrm{CO}_{2}\right]^{-}$ & Protocatechuic acid ${ }^{a}$ & RR, RS \\
\hline 10 & 5.23 & $\mathrm{C}_{6} \mathrm{H}_{8} \mathrm{O}_{7}$ & $\begin{array}{c}193.03373 \\
(-2.8) \\
\end{array}$ & $\begin{array}{c}191.02033 \\
(+3.1)\end{array}$ & & $\begin{array}{l}155.0029\left[\mathrm{M}-\mathrm{H}-2 \mathrm{H}_{2} \mathrm{O}\right]^{-} \\
111.0079\left[\mathrm{M}-\mathrm{H}-2 \mathrm{H}_{2} \mathrm{O}-\mathrm{CO}_{2}\right]^{-}\end{array}$ & Citric acid ${ }^{\text {a }}$ & RR, RS \\
\hline 11 & 5.65 & $\mathrm{C}_{9} \mathrm{H}_{11} \mathrm{NO}_{3}$ & $\begin{array}{l}182.08028 \\
(-4.9)\end{array}$ & & $\begin{array}{l}\left.\text { 147.0444 [M }+\mathrm{H}-\mathrm{H}_{2} \mathrm{O}-\mathrm{NH}_{3}\right]^{+} \\
136.0761\left[\mathrm{M}+\mathrm{H}-\mathrm{HCOOH}^{+},\right. \\
119.0493\left[\mathrm{M}+\mathrm{H}-\mathrm{C}_{2} \mathrm{H}_{3} \mathrm{NO}_{2}\right]^{+} \\
91.0992\left[\mathrm{M}+\mathrm{H}-\mathrm{C}_{2} \mathrm{H}_{3} \mathrm{NO}_{2}-\mathrm{H}_{2} \mathrm{O}\right]^{+}\end{array}$ & & Tyrosine $^{a}$ & RR, RS \\
\hline 12 & 5.65 & $\mathrm{C}_{9} \mathrm{H}_{8} \mathrm{O}_{3}$ & $\begin{array}{c}165.05427 \\
(-2.1)\end{array}$ & $\begin{array}{c}163.04076 \\
(+4.2)\end{array}$ & $\begin{array}{l}119.0467[\mathrm{M}+\mathrm{H}-\mathrm{HCOOH}]^{+} \\
91.0563\left[\mathrm{M}+\mathrm{H}-\mathrm{HCOOH}-\mathrm{H}_{2} \mathrm{O}\right]^{+}\end{array}$ & & $p$-Coumaric acid ${ }^{\text {a }}$ & RR, RS \\
\hline 13 & 5.73 & $\mathrm{C}_{6} \mathrm{H}_{13} \mathrm{NO}_{2}$ & $\begin{array}{c}132.10173 \\
(-1.3)\end{array}$ & $\begin{array}{c}130.08736 \\
(0)\end{array}$ & $\begin{array}{l}86.0981[\mathrm{M}+\mathrm{H}-\mathrm{HCOOH}]^{+} \\
69.0722\left[\mathrm{M}+\mathrm{H}-\mathrm{HCOOH}-\mathrm{NH}_{3}\right]^{+}\end{array}$ & & Isoleucine $^{\mathrm{a}}$ & RR, RS \\
\hline 14 & 6.13 & $\mathrm{C}_{6} \mathrm{H}_{13} \mathrm{NO}_{2}$ & $\begin{array}{c}132.10198 \\
(+0.6)\end{array}$ & $\begin{array}{c}130.08852 \\
(+9.0)\end{array}$ & $\begin{array}{l}86.0982[\mathrm{M}+\mathrm{H}-\mathrm{HCOOH}]^{+} \\
69.0720\left[\mathrm{M}+\mathrm{H}-\mathrm{HCOOH}-\mathrm{NH}_{3}\right]^{+}\end{array}$ & & Leucine $^{a}$ & RR, RS \\
\hline 15 & 6.99 & $\mathrm{C}_{8} \mathrm{H}_{8} \mathrm{O}_{4}$ & $\begin{array}{c}169.04931 \\
(-1.3)\end{array}$ & $\begin{array}{c}167.03526 \\
(+1.7)\end{array}$ & $\begin{array}{l}150.9672\left[\mathrm{M}+\mathrm{H}-\mathrm{H}_{2} \mathrm{O}\right]^{+} \\
95.0136\end{array}$ & $109.0268[\mathrm{M}-\mathrm{H}-\mathrm{CO}-\mathrm{HCOH}]^{-}$ & Vanillin $^{a}$ & $\mathrm{RR}$ \\
\hline 16 & 7.72 & $\mathrm{C}_{7} \mathrm{H}_{6} \mathrm{O}_{5}$ & $\begin{array}{c}171.02825 \\
(-3.2)\end{array}$ & $\begin{array}{c}169.0144 \\
(+0.9)\end{array}$ & $\begin{array}{l}\text { 139.0017, } \\
111.0063\end{array}$ & $125.0238\left[\mathrm{M}-\mathrm{H}-\mathrm{CO}_{2}\right]^{-}$ & Gallic acid $^{a}$ & RR, RS \\
\hline
\end{tabular}


Table 2. Cont.

\begin{tabular}{|c|c|c|c|c|c|c|c|c|}
\hline No. & $\begin{array}{c}\mathrm{Rt} \\
(\mathrm{min})\end{array}$ & $\begin{array}{l}\text { Molecular } \\
\text { Formula }\end{array}$ & {$[\mathrm{M}+\mathrm{H}]^{+}$} & {$[\mathbf{M}-\mathbf{H}]^{-}$} & $\begin{array}{l}\text { Major Fragment Ions } \\
\text { in Positive Mode }\end{array}$ & $\begin{array}{l}\text { Major Fragment Ions } \\
\text { in Negative Mode }\end{array}$ & Identification & Source \\
\hline 17 & 8.09 & $\mathrm{C}_{9} \mathrm{H}_{11} \mathrm{NO}_{2}$ & $\begin{array}{c}166.08574 \\
(-3.1)\end{array}$ & $\begin{array}{c}164.07209 \\
(+2.4)\end{array}$ & $\begin{array}{l}120.0809[\mathrm{M}+\mathrm{H}-\mathrm{HCOOH}]^{+}, 103.0548 \\
{\left[\mathrm{M}+\mathrm{H}-\mathrm{HCOOH}-\mathrm{NH}_{3}\right]^{+}}\end{array}$ & $\begin{array}{l}147.0454\left[\mathrm{M}-\mathrm{H}-\mathrm{NH}_{3}\right]^{-} \\
120.0444\left[\mathrm{M}-\mathrm{H}-\mathrm{CO}_{2}\right]^{-}\end{array}$ & Phenylalanine $^{\mathrm{a}}$ & RR, RS \\
\hline 18 & 9.27 & $\mathrm{C}_{15} \mathrm{H}_{14} \mathrm{O}_{7}$ & $\begin{array}{c}307.08106 \\
(-0.6)\end{array}$ & $\begin{array}{c}305.06686 \\
(+0.6)\end{array}$ & & $125.0234\left[\mathrm{M}-\mathrm{H}-\mathrm{C}_{8} \mathrm{H}_{8} \mathrm{O}_{4}\right]^{-}$ & Epigallocatechin & RS \\
\hline 19 & 10.07 & $\mathrm{C}_{9} \mathrm{H}_{10} \mathrm{O}_{5}$ & & $\begin{array}{c}197.04591 \\
(+1.8)\end{array}$ & & $\begin{array}{l}151.0430\left[\mathrm{M}-\mathrm{H}-\mathrm{HCOOH}^{-}\right. \\
125.0248\left[\mathrm{M}-\mathrm{H}-\mathrm{CO}_{2}-\mathrm{H}_{2} \mathrm{O}\right]^{-}\end{array}$ & Syringic acid ${ }^{a}$ & $\mathrm{RR}$ \\
\hline 20 & 10.14 & $\mathrm{C}_{11} \mathrm{H}_{12} \mathrm{~N}_{2} \mathrm{O}_{2}$ & $\begin{array}{l}205.09718 \\
(+0.1)\end{array}$ & $\begin{array}{l}203.08256 \\
(-0.2)\end{array}$ & $\begin{array}{l}188.0691\left[\mathrm{M}+\mathrm{H}-\mathrm{NH}_{3}\right]^{+}, \\
170.0595\left[\mathrm{M}+\mathrm{H}-\mathrm{NH}_{3}-\mathrm{H}_{2} \mathrm{O}\right]^{+}, \\
146.0593 \\
118.0648\end{array}$ & $116.0507\left[\mathrm{M}-\mathrm{H}-\mathrm{C}_{3} \mathrm{H}_{7} \mathrm{NO}_{2}\right]^{-}$ & Tryptophan $^{\text {a }}$ & RR, RS \\
\hline 21 & 10.40 & $\mathrm{C}_{30} \mathrm{H}_{26} \mathrm{O}_{12}$ & $\begin{array}{l}579.14939 \\
(-0.5)\end{array}$ & $\begin{array}{c}577.13504 \\
(-0.2)\end{array}$ & $\begin{array}{l}\left.\text { 427.1022 [M+H }-\mathrm{C}_{8} \mathrm{H}_{8} \mathrm{O}_{3}\right]^{+} \\
409.0898\left[\mathrm{M}+\mathrm{H}-\mathrm{C}_{8} \mathrm{H}_{8} \mathrm{O}_{3}-\mathrm{H}_{2} \mathrm{O}\right]^{+} \\
287.0554\left[\mathrm{M}+\mathrm{H}-\mathrm{C}_{15} \mathrm{H}_{16} \mathrm{O}_{6}\right]+\end{array}$ & $\begin{array}{l}\text { 451.1050, } \\
\begin{array}{l}425.0893\left[\mathrm{M}-\mathrm{H}-\mathrm{C}_{8} \mathrm{H}_{8} \mathrm{O}_{3}\right]^{-} \\
407.0783\left[\mathrm{M}-\mathrm{H}-\mathrm{C}_{8} \mathrm{H}_{8} \mathrm{O}_{3}-\mathrm{H}_{2} \mathrm{O}\right]^{-} \\
289.0729\left[\mathrm{M}-\mathrm{H}-\mathrm{C}_{15} \mathrm{H}_{12} \mathrm{O}_{6}\right]^{-}\end{array}\end{array}$ & Procyanidin B1 & RR, RS \\
\hline 22 & 10.54 & $\mathrm{C}_{9} \mathrm{H}_{10} \mathrm{O}_{4}$ & $\begin{array}{c}183.06502 \\
(-0.9)\end{array}$ & $\begin{array}{l}181.05076 \\
(+0.7)\end{array}$ & & $\begin{array}{l}163.0367\left[\mathrm{M}-\mathrm{H}-\mathrm{H}_{2} \mathrm{O}\right]^{-} \\
135.0449\left[\mathrm{M}-\mathrm{H}-\mathrm{H}_{2} \mathrm{O}-\mathrm{CO}^{-}\right. \\
119.0495\left[\mathrm{M}-\mathrm{H}-\mathrm{CH}_{2} \mathrm{O}-\mathrm{CH}_{4} \mathrm{O}\right]^{-}\end{array}$ & Syringaldehyde & $\mathrm{RR}$ \\
\hline 23 & 10.72 & $\mathrm{C}_{13} \mathrm{H}_{16} \mathrm{O}_{8}$ & & $\begin{array}{c}299.0775 \\
(+0.9)\end{array}$ & & $\begin{array}{l}137.0241\left[\mathrm{M}-\mathrm{H}-\mathrm{glc}^{-}\right. \\
93.0358\left[\mathrm{M}-\mathrm{H}-\mathrm{glc}-\mathrm{CO}_{2}\right]^{-}\end{array}$ & $\begin{array}{l}\text { 4-Hydroxybenzoic } \\
\text { acid-4-O-glucopyranoside }\end{array}$ & RS \\
\hline 24 & 11.06 & $\mathrm{C}_{30} \mathrm{H}_{26} \mathrm{O}_{12}$ & $\begin{array}{l}579.14973 \\
\quad(0)\end{array}$ & $\begin{array}{l}577.13567 \\
\quad(+0.9)\end{array}$ & $\begin{array}{l}\text { 453.1169, } \\
427.1037, \\
409.0923 \\
301.0721 \\
287.0554\end{array}$ & $289.0730\left[\mathrm{M}-\mathrm{H}-\mathrm{C}_{15} \mathrm{H}_{12} \mathrm{O}_{6}\right]^{-}$ & Procyanidin B2 & RR, RS \\
\hline 25 & 11.73 & $\mathrm{C}_{7} \mathrm{H}_{6} \mathrm{O}_{3}$ & $\begin{array}{c}139.03867 \\
(-2.2)\end{array}$ & $\begin{array}{c}137.02506 \\
(+4.7)\end{array}$ & $\begin{array}{l}111.0440\left[\mathrm{M}+\mathrm{H}-\mathrm{H}_{2} \mathrm{O}\right]^{+} \\
95.0135\left[\mathrm{M}+\mathrm{H}-\mathrm{CO}_{2}\right]^{+}\end{array}$ & $93.0351\left[\mathrm{M}-\mathrm{H}-\mathrm{CO}_{2}\right]^{-}$ & 4-Hydroxybenzoic acid ${ }^{\text {a }}$ & RR, RS \\
\hline 26 & 12.39 & $\mathrm{C}_{21} \mathrm{H}_{26} \mathrm{O}_{8}$ & $\begin{array}{c}407.16816 \\
(-4.6)\end{array}$ & & $245.0449[\mathrm{M}+\mathrm{H}-\mathrm{glc}]^{+}$ & & $\begin{array}{l}\text { Erythro-guaiacylglycerol } \beta \text {-sinapyl } \\
\text { ether or threo-guaiacylglycerol } \\
\beta \text {-sinapyl ether }\end{array}$ & RR, RS \\
\hline 27 & 12.41 & $\mathrm{C}_{30} \mathrm{H}_{26} \mathrm{O}_{11}$ & & $\begin{array}{c}561.14044 \\
(+0.4)\end{array}$ & & $\begin{array}{l}407.0756\left[\mathrm{M}-\mathrm{H}-\mathrm{C}_{8} \mathrm{H}_{10} \mathrm{O}_{3}\right]^{-}, \\
289.0718\left[\mathrm{M}-\mathrm{H}-\mathrm{C}_{15} \mathrm{H}_{12} \mathrm{O}_{5}\right]^{-} \\
273.07006\left[\mathrm{M}-\mathrm{H}-\mathrm{C}_{15} \mathrm{H}_{12} \mathrm{O}_{6}\right]^{-}\end{array}$ & Fisetinidol-( $4 \alpha, 8)$-catechin & RS \\
\hline 28 & 13.01 & $\mathrm{C}_{9} \mathrm{H}_{8} \mathrm{O}_{4}$ & & $\begin{array}{c}179.03508 \\
(+0.5)\end{array}$ & & $135.0446\left[\mathrm{M}-\mathrm{H}-\mathrm{CO}_{2}\right]^{-}$ & Caffeic acid ${ }^{\text {a }}$ & RR, RS \\
\hline 29 & 13.29 & $\mathrm{C}_{30} \mathrm{H}_{26} \mathrm{O}_{12}$ & $\begin{array}{c}579.14959 \\
(-0.2)\end{array}$ & $\begin{array}{l}577.13543 \\
(+0.5)\end{array}$ & $\begin{array}{l}\text { 439.1030, } \\
\text { 427.1038 }\left[\mathrm{M}+\mathrm{H}-\mathrm{C}_{8} \mathrm{H}_{8} \mathrm{O}_{3}\right]^{+} \\
\text {409.0909 }\left[\mathrm{M}+\mathrm{H}-\mathrm{C}_{8} \mathrm{H}_{8} \mathrm{O}_{3}-\mathrm{H}_{2} \mathrm{O}\right]^{+} \\
301.0738 \\
287.0554 \\
271.0620\end{array}$ & 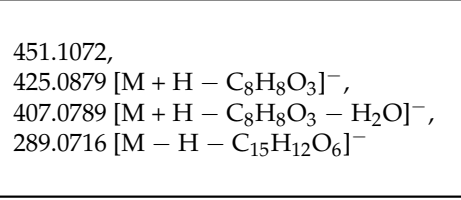 & Procyanidin B3 & RR, RS \\
\hline
\end{tabular}


Table 2. Cont

\begin{tabular}{|c|c|c|c|c|c|c|c|c|}
\hline No. & $\begin{array}{c}\mathbf{R t} \\
(\min )\end{array}$ & $\begin{array}{l}\text { Molecular } \\
\text { Formula }\end{array}$ & {$[\mathrm{M}+\mathbf{H}]^{+}$} & {$[\mathbf{M}-\mathbf{H}]^{-}$} & $\begin{array}{l}\text { Major Fragment Ions } \\
\text { in Positive Mode }\end{array}$ & $\begin{array}{l}\text { Major Fragment Ions } \\
\text { in Negative Mode }\end{array}$ & Identification & Source \\
\hline 30 & 14.06 & $\mathrm{C}_{27} \mathrm{H}_{30} \mathrm{O}_{16}$ & $\begin{array}{c}611.16016 \\
(-0.8)\end{array}$ & $\begin{array}{c}609.14646 \\
(+0.6)\end{array}$ & $303.0460\left[\mathrm{M}+\mathrm{H}-\right.$ rutinose $^{+}$ & $\begin{array}{l}301.0333\left[\mathrm{M}-\mathrm{H}-\text { rui }^{-},\right. \\
271.0238\left[\mathrm{M}-\mathrm{H}-\text { rui }-\mathrm{CH}_{2} \mathrm{O}\right]^{-}\end{array}$ & Rutin $^{a}$ & $\mathrm{RR}, \mathrm{RS}$ \\
\hline 31 & 14.72 & $\mathrm{C}_{21} \mathrm{H}_{20} \mathrm{O}_{12}$ & $\begin{array}{l}465.10249 \\
(-0.6)\end{array}$ & $\begin{array}{l}463.08802 \\
(-0.4)\end{array}$ & $303.0504[\mathrm{M}+\mathrm{H}-\mathrm{glc}]^{+}$ & $\begin{array}{l}301.0347\left[\mathrm{M}-\mathrm{H}-\mathrm{glc}^{-}\right. \\
271.0260 \\
255.0292 \\
151.0027\end{array}$ & Isoquercitrin $^{\text {a }}$ & $\mathrm{RR}, \mathrm{RS}$ \\
\hline 32 & 15.18 & $\mathrm{C}_{27} \mathrm{H}_{28} \mathrm{O}_{16}$ & $\begin{array}{c}609.14377 \\
(-2.0)\end{array}$ & $\begin{array}{l}607.13045 \\
(0)\end{array}$ & 303.0491 & $\begin{array}{l}463.0839 \\
301.0352\end{array}$ & $\begin{array}{l}\text { Quercetin 3-O-[(X-O-3-hydroxy-3- } \\
\text { methylglutaryl)- } \beta \text {-glucoside }]\end{array}$ & RR, RS \\
\hline 33 & 15.23 & $\mathrm{C}_{22} \mathrm{H}_{26} \mathrm{O}_{8}$ & & $\begin{array}{c}417.15509 \\
(-1.0)\end{array}$ & & $181.0482\left[\mathrm{M}-\mathrm{H}-\mathrm{C}_{13} \mathrm{H}_{16} \mathrm{O}_{4}\right]^{-}$ & Diasyringaresinol & RR \\
\hline 34 & 15.57 & $\mathrm{C}_{20} \mathrm{H}_{18} \mathrm{O}_{11}$ & $\begin{array}{c}435.09253 \\
(+0.8)\end{array}$ & $\begin{array}{c}433.07753 \\
(-0.2)\end{array}$ & $303.0508\left[\mathrm{M}+\mathrm{H}-\mathrm{C}_{5} \mathrm{H}_{10} \mathrm{O}_{4}\right]^{+}$ & $301.0358\left[\mathrm{M}-\mathrm{H}-\mathrm{C}_{5} \mathrm{H}_{10} \mathrm{O}_{4}\right]^{-}$ & Quercetin-3-O-D-xyloside & RR, RS \\
\hline 35 & 15.77 & $\mathrm{C}_{21} \mathrm{H}_{20} \mathrm{O}_{11}$ & $\begin{array}{l}449.10749 \\
\quad(-0.8)\end{array}$ & $\begin{array}{l}447.09296 \\
\quad(-0.7)\end{array}$ & $\begin{array}{l}\left.\text { 303.0846 [M + H }-\mathrm{C}_{6} \mathrm{H}_{12} \mathrm{O}_{4}\right]^{+}, \\
151.0380 \\
123.0429\end{array}$ & $\begin{array}{l}300.9984\left[\mathrm{M}-\mathrm{H}-\mathrm{C}_{6} \mathrm{H}_{12} \mathrm{O}_{4}\right]^{-} \\
285.0406\end{array}$ & Quercitrin $^{\text {a }}$ & $\mathrm{RR}, \mathrm{RS}$ \\
\hline 36 & 15.94 & $\mathrm{C}_{27} \mathrm{H}_{28} \mathrm{O}_{15}$ & $\begin{array}{l}593.14886 \\
(-2.1)\end{array}$ & $\begin{array}{c}591.13566 \\
(+0.2)\end{array}$ & 287.0543 & $\begin{array}{l}529.1312, \\
489.1046, \\
447.0920 \\
285.0309\end{array}$ & $\begin{array}{l}\text { Kaempferol 3-O-[(X-O-3- } \\
\text { hydroxy-3-methylglutaryl)- } \\
\beta \text {-galactoside] }\end{array}$ & $\mathrm{RR}, \mathrm{RS}$ \\
\hline 37 & 16.28 & $\mathrm{C}_{20} \mathrm{H}_{22} \mathrm{O}_{8}$ & & $\begin{array}{l}389.12421 \\
\quad(0)\end{array}$ & & $227.0712\left[\mathrm{M}-\mathrm{H}-\mathrm{glc}^{-}\right.$ & Piceid & RR, RS \\
\hline 38 & 16.36 & $\mathrm{C}_{27} \mathrm{H}_{28} \mathrm{O}_{15}$ & $\begin{array}{l}593.14843 \\
(-2.8)\end{array}$ & $\begin{array}{l}591.13617 \\
(+1.1)\end{array}$ & 287.0544 & $\begin{array}{l}529.1236 \\
489.1044 \\
447.0936 \\
285.0407\end{array}$ & $\begin{array}{l}\text { Kaempferol 3-O-[(X-O-3- } \\
\text { hydroxy-3-methylglutaryl)- } \\
\beta \text {-glucoside }]\end{array}$ & $\mathrm{RR}, \mathrm{RS}$ \\
\hline 39 & 16.83 & $\mathrm{C}_{21} \mathrm{H}_{24} \mathrm{O}_{10}$ & & $\begin{array}{l}435.12995 \\
(0.6)\end{array}$ & & $\begin{array}{l}273.0781\left[\mathrm{M}-\mathrm{H}-\mathrm{glc}^{-},\right. \\
167.0349\end{array}$ & Phloridzin & $\mathrm{RR}$ \\
\hline 40 & 17.21 & $\mathrm{C}_{15} \mathrm{H}_{14} \mathrm{O}_{2}$ & $\begin{array}{c}227.10655 \\
(-0.4)\end{array}$ & & $\begin{array}{l}\left.\text { 197.0650 [M + H }-\mathrm{CH}_{2} \mathrm{O}\right]^{+} \\
185.0997\left[\mathrm{M}+\mathrm{H}-\mathrm{CH}_{2} \mathrm{O}-\mathrm{H}_{2} \mathrm{O}\right]^{+}\end{array}$ & & 3-Methoxy-5-hydroxy-stilbene & $\mathrm{RR}, \mathrm{RS}$ \\
\hline 41 & 17.35 & $\mathrm{C}_{21} \mathrm{H}_{26} \mathrm{O}_{8}$ & $\begin{array}{l}407.16945 \\
\quad(-1.5)\end{array}$ & & $\begin{array}{l}389.1582\left[\mathrm{M}+\mathrm{H}-\mathrm{H}_{2} \mathrm{O}\right]^{+} \\
371.1472\left[\mathrm{M}+\mathrm{H}-2 \mathrm{H}_{2} \mathrm{O}\right]^{+} \\
245.1159\left[\mathrm{M}+\mathrm{H}-\mathrm{glc}^{+},\right. \\
215.1062, \\
199.1114\end{array}$ & & $\begin{array}{l}\text { Erythro-guaiacylglycerol } \beta \text {-sinapyl } \\
\text { ether or threo-guaiacylglycerol } \\
\beta \text {-sinapyl ether }\end{array}$ & $\mathrm{RR}, \mathrm{RS}$ \\
\hline 42 & 18.53 & $\mathrm{C}_{20} \mathrm{H}_{22} \mathrm{O}_{6}$ & $\begin{array}{l}359.14831 \\
(-1.7)\end{array}$ & $\begin{array}{l}357.13435 \\
\quad(0)\end{array}$ & $\begin{array}{l}\text { 327.1216 [M- }-\mathrm{H}-\mathrm{CH}_{4} \mathrm{O}^{-} \\
\text {313.1057 } \\
\text { 253.0856 }-\mathrm{H}-\mathrm{H}_{2} \mathrm{O}-\mathrm{CO}^{-}\end{array}$ & & Pinoresinol & RR, RS \\
\hline
\end{tabular}


Table 2. Cont.

\begin{tabular}{|c|c|c|c|c|c|c|c|c|}
\hline No. & $\begin{array}{c}\mathbf{R t} \\
\text { (min) }\end{array}$ & $\begin{array}{c}\text { Molecular } \\
\text { Formula }\end{array}$ & {$[\mathbf{M}+\mathbf{H}]^{+}$} & {$[\mathbf{M}-\mathbf{H}]^{-}$} & $\begin{array}{l}\text { Major Fragment Ions } \\
\text { in Positive Mode }\end{array}$ & $\begin{array}{l}\text { Major Fragment Ions } \\
\text { in Negative Mode }\end{array}$ & Identification & Source \\
\hline 43 & 18.68 & $\mathrm{C}_{36} \mathrm{H}_{58} \mathrm{O}_{11}$ & & $\begin{array}{c}665.39109 \\
(+0.7)\end{array}$ & & $\begin{array}{l}619.3935\left[\mathrm{M}-\mathrm{H}-2 \mathrm{H}_{2} \mathrm{O}\right]^{-}, \\
485.3284\left[\mathrm{M}-\mathrm{H}-\mathrm{glc}-\mathrm{H}_{2} \mathrm{O}\right]^{-}, \\
441.3399\left[\mathrm{M}-\mathrm{H}-\mathrm{glc}-\mathrm{H}_{2} \mathrm{O}-\mathrm{CO}_{2}\right]^{-}, \\
357.2748\end{array}$ & $\begin{array}{l}\text { Polygalacic acid } \\
\text { 3-O- } \beta \text {-D-glucopyranoside }\end{array}$ & $\mathrm{RR}, \mathrm{RS}$ \\
\hline 44 & 18.9 & $\mathrm{C}_{15} \mathrm{H}_{10} \mathrm{O}_{7}$ & $\begin{array}{c}303.0497 \\
(-0.7)\end{array}$ & $\begin{array}{c}301.03548 \\
\quad(+0.4)\end{array}$ & $\begin{array}{l}\text { 257.0431, } \\
229.0497, \\
201.0540, \\
165.0182, \\
153.0181 \\
137.0230\end{array}$ & $178.9987,151.0038,121.0290$ & Quercetin $^{a}$ & $\mathrm{RR}, \mathrm{RS}$ \\
\hline 45 & 19.38 & $\mathrm{C}_{36} \mathrm{H}_{58} \mathrm{O}_{11}$ & & $\begin{array}{c}665.39114 \\
(+0.8)\end{array}$ & & $\begin{array}{l}485.3272\left[\mathrm{M}-\mathrm{H}-\mathrm{glc}-\mathrm{H}_{2} \mathrm{O}\right]^{-} \\
467.3211\left[\mathrm{M}-\mathrm{H}-\mathrm{glc}-2 \mathrm{H}_{2} \mathrm{O}\right]^{-} \\
351.2653\end{array}$ & $\begin{array}{l}19 \alpha \text {-hydroxyasiatic } \\
\text { acid-28-O- } \beta \text {-D-glucopyranoside }\end{array}$ & RR, RSS \\
\hline 46 & 20.39 & $\mathrm{C}_{36} \mathrm{H}_{58} \mathrm{O}_{10}$ & & $\begin{array}{c}649.39552 \\
(-0.3) \\
\end{array}$ & & $\begin{array}{l}487.3427\left[\mathrm{M}-\mathrm{H}-\mathrm{glc}^{-}\right. \\
469.3319\left[\mathrm{M}-\mathrm{H}-\mathrm{glc}-\mathrm{H}_{2} \mathrm{O}\right]^{-}\end{array}$ & Kajiichigoside F1 & $\mathrm{RR}, \mathrm{RS}$ \\
\hline 47 & 20.64 & $\mathrm{C}_{15} \mathrm{H}_{10} \mathrm{O}_{6}$ & $\begin{array}{c}287.05474 \\
(-1.0) \\
\end{array}$ & & $165.0133,153.0202$ & & Kaempferol $^{\text {a }}$ & RR, RSS \\
\hline 48 & 22.10 & $\mathrm{C}_{15} \mathrm{H}_{12} \mathrm{O}_{5}$ & $\begin{array}{c}273.07551 \\
(-0.9)\end{array}$ & & 153.0181 & 151.003 & Dihydroapigenin & $R R, R S$ \\
\hline 49 & 22.95 & $\mathrm{C}_{15} \mathrm{H}_{10} \mathrm{O}_{6}$ & $\begin{array}{c}287.05465 \\
(-1.3)\end{array}$ & $\begin{array}{c}285.04066 \\
(+0.7)\end{array}$ & $\begin{array}{l}268.9789, \\
153.0188 \\
121.0251\end{array}$ & $\begin{array}{l}229.0563 \\
187.0393 \\
169.0649 \\
143.0520\end{array}$ & Luteolin $^{a}$ & $\mathrm{RR}, \mathrm{RS}$ \\
\hline 50 & 26.48 & $\mathrm{C} 30 \mathrm{H} 48 \mathrm{O} 6$ & & $\begin{array}{c}503.33793 \\
(+0.2)\end{array}$ & & $\begin{array}{l}485.3320\left[\mathrm{M}-\mathrm{H}-\mathrm{H}_{2} \mathrm{O}\right]- \\
439.3229\left[\mathrm{M}-\mathrm{H}-\mathrm{HCOOH}-\mathrm{H}_{2} \mathrm{O}\right]- \\
421.3141\left[\mathrm{M}-\mathrm{H}-\mathrm{HCOOH}-2 \mathrm{H}_{2} \mathrm{O}\right]- \\
225.1623\end{array}$ & 1-Hydroxyeuscaphic acid & $\mathrm{RR}, \mathrm{RS}$ \\
\hline 51 & 27.25 & $\mathrm{C} 30 \mathrm{H} 46 \mathrm{O} 5$ & $\begin{array}{c}487.34155 \\
(-0.5)\end{array}$ & $\begin{array}{l}485.32722 \\
(-0.1)\end{array}$ & $\begin{array}{l}451.3208\left[\mathrm{M}+\mathrm{H}-2 \mathrm{H}_{2} \mathrm{O}\right]+ \\
405.3170\left[\mathrm{M}+\mathrm{H}-2 \mathrm{H}_{2} \mathrm{O}-\mathrm{HCOOH}\right]+ \\
223.1604 \\
199.1448 \\
187.1454\end{array}$ & $\begin{array}{l}467.3176 \\
425.23124 \\
375.3047 \\
321.2564 \\
257.2382 \\
\end{array}$ & $\begin{array}{l}2 \alpha, 19 \alpha \text {-Dihydroxy-3-oxo-urs- } \\
12 \text {-en-28-oic acid isomer }\end{array}$ & $\mathrm{RR}, \mathrm{RS}$ \\
\hline 52 & 29.27 & $\mathrm{C} 30 \mathrm{H} 48 \mathrm{O} 5$ & $\begin{array}{l}489.35729 \\
(-0.3)\end{array}$ & $\begin{array}{l}487.34276 \\
\quad(-0.3)\end{array}$ & $\begin{array}{l}407.3131\left[\mathrm{M}+\mathrm{H}-2 \mathrm{H}_{2} \mathrm{O}-\mathrm{HCOOH}\right]+ \\
\text { 207.1733, } \\
201.1624\end{array}$ & $\begin{array}{l}469.3302\left[\mathrm{M}-\mathrm{H}-\mathrm{H}_{2} \mathrm{O}\right]- \\
443.3533\left[\mathrm{M}-\mathrm{H}-\mathrm{CO}_{2}\right]- \\
427.3205\left[\mathrm{M}-\mathrm{H}-\mathrm{H}_{2} \mathrm{O}-\mathrm{CO}_{2}\right]- \\
371.2911\end{array}$ & Euscaphic acid & RR, RSS \\
\hline 53 & 33.05 & $\mathrm{C} 30 \mathrm{H} 46 \mathrm{O} 5$ & $\begin{array}{c}487.34155 \\
(-0.5)\end{array}$ & $\begin{array}{c}485.32728 \\
(+0.1)\end{array}$ & $\begin{array}{l}451.3208\left[\mathrm{M}+\mathrm{H}-2 \mathrm{H}_{2} \mathrm{O}\right]+ \\
405.3170\left[\mathrm{M}+\mathrm{H}-2 \mathrm{H}_{2} \mathrm{O}-\mathrm{HCOOH}\right]+, \\
223.1604 \\
199.1448 \\
187.1454\end{array}$ & $\begin{array}{l}467.3186\left[\mathrm{M}-\mathrm{H}-\mathrm{H}_{2} \mathrm{O}\right]- \\
441.3415\left[\mathrm{M}-\mathrm{H}-\mathrm{CO}_{2}\right]-, \\
423.3280\left[\mathrm{M}-\mathrm{H}-\mathrm{H}_{2} \mathrm{O}-\mathrm{CO}_{2}\right]- \\
393.3161\end{array}$ & 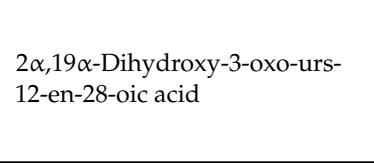 & $\mathrm{RR}, \mathrm{RS}$ \\
\hline
\end{tabular}


Table 2. Cont.

\begin{tabular}{|c|c|c|c|c|c|c|c|c|}
\hline No. & $\begin{array}{c}\mathrm{Rt} \\
(\mathrm{min})\end{array}$ & $\begin{array}{l}\text { Molecular } \\
\text { Formula }\end{array}$ & {$[\mathrm{M}+\mathrm{H}]^{+}$} & {$[\mathbf{M}-\mathbf{H}]^{-}$} & $\begin{array}{l}\text { Major Fragment Ions } \\
\text { in Positive Mode }\end{array}$ & $\begin{array}{l}\text { Major Fragment Ions } \\
\text { in Negative Mode }\end{array}$ & Identification & Source \\
\hline 54 & 34.87 & $\mathrm{C} 30 \mathrm{H} 48 \mathrm{O} 4$ & & $\begin{array}{c}471.34821 \\
(+0.5)\end{array}$ & & $\begin{array}{l}453.3398\left[\mathrm{M}-\mathrm{H}-\mathrm{H}_{2} \mathrm{O}\right]- \\
423.3270 \\
407.3350 \\
377.2865\end{array}$ & Pomolic acid or isomer & $\mathrm{RR}, \mathrm{RS}$ \\
\hline 55 & 35.53 & $\mathrm{C} 30 \mathrm{H} 48 \mathrm{O} 4$ & & $471.348(0)$ & & $\begin{array}{l}453.3350\left[\mathrm{M}-\mathrm{H}-\mathrm{H}_{2} \mathrm{O}\right]- \\
\text { 407.3315, } \\
377.2888\end{array}$ & Pomolic acid or isomer & $\mathrm{RR}, \mathrm{RS}$ \\
\hline 56 & 35.56 & $\mathrm{C} 30 \mathrm{H} 46 \mathrm{O} 4$ & & $\begin{array}{l}469.33187 \\
(-1.0)\end{array}$ & & $\begin{array}{l}451.3237\left[\mathrm{M}-\mathrm{H}-\mathrm{H}_{2} \mathrm{O}\right]- \\
407.3304\left[\mathrm{M}-\mathrm{H}-\mathrm{H}_{2} \mathrm{O}-\mathrm{CO}_{2}\right]- \\
377.3222\end{array}$ & $\begin{array}{l}2 \alpha, 3 \beta \text {-Dihydroxylup-20(29)- } \\
\text { en-28-oic acid }\end{array}$ & $\mathrm{RR}, \mathrm{RS}$ \\
\hline 57 & 40.72 & $\mathrm{C} 18 \mathrm{H} 30 \mathrm{O} 2$ & $\begin{array}{c}279.2318 \\
(-0.2)\end{array}$ & & & & $9,12,15$-Octadecatrienoic acid ${ }^{a}$ & RS \\
\hline 58 & 42.58 & $\mathrm{C} 30 \mathrm{H} 48 \mathrm{O} 3$ & $\begin{array}{c}457.36755 \\
(-0.2)\end{array}$ & $\begin{array}{c}455.35307 \\
(-0.6)\end{array}$ & & 407.3335 [M - H - HCOOH]- & Ursolic acid a $^{\text {a }}$ & $\mathrm{RR}, \mathrm{RS}$ \\
\hline 59 & 42.68 & $\mathrm{C} 18 \mathrm{H} 32 \mathrm{O} 2$ & $\begin{array}{c}281.24735 \\
(-0.6)\end{array}$ & $\begin{array}{c}279.23322 \\
(+1.0)\end{array}$ & & $261.2124\left[\mathrm{M}-\mathrm{H}-\mathrm{H}_{2} \mathrm{O}\right]-$ & 9,12-Octadecadienoic acid a & $\mathrm{RR}, \mathrm{RS}$ \\
\hline
\end{tabular}

${ }^{a}$ Compound was confirmed by reference standard. RR: R. roxburghii; RS: R. sterilis. 
Amino acids: Nine amino acids identified in the two fruits have been confirmed with reference standards, and they were serine (2), arginine (3), proline (4), valine (7), tyrosine (11), isoleucine (13), leucine (14), phenylalanine (17) and tryptophan (20) [18].

Phenylpropanoid derivatives: Peaks 26 and 41, a pair of isomers, gave a $[\mathrm{M}+\mathrm{H}]^{+}$ion at $m / z$ $407.16\left(\mathrm{C}_{21} \mathrm{H}_{26} \mathrm{O}_{8}\right)$ and the fragment ion of $m / z 245,162 \mathrm{Da}$ lost from the precursor ion, corresponding to a glucose group. Thus, they were identified as erythro-guaiacylglycerol $\beta$-sinapyl ether and threo-guaiacylglycerol $\beta$-sinapyl ether, but the configuration of the chiral carbon cannot be confirmed without NMR [26]. Peak 33 gave a $[\mathrm{M}-\mathrm{H}]^{-}$ion at $m / z 417.15509$, with a molecular formula $\mathrm{C}_{22} \mathrm{H}_{26} \mathrm{O}_{8}$ by TOF-MS. The fragment ion at $m / z 181$ was produced by the dissociation of the furan ring; it therefore was identified as diasyringaresinol [33]. Peak 39 showed a $[\mathrm{M}-\mathrm{H}]^{-}$ion at $m / z 435.12995\left(\mathrm{C}_{21} \mathrm{H}_{24} \mathrm{O}_{10}\right)$. The fragment ion of $m / z 273$ unequivocally illustrated the existence of a glucose group. Thus it was tentatively identified as phlorizin [36]. Peak 42 with a protonated $[\mathrm{M}+\mathrm{H}]^{+}$ion at $m / z 359.14831$ $\left(\mathrm{C}_{20} \mathrm{H}_{22} \mathrm{O}_{6}\right)$ was identified as pinoresinol compared with a previous report [36].

Condensed tannins: In the MS spectra, four condensed tannins were identified on the basis of a fragmentation pattern with successive loss of $288 \mathrm{Da}$ corresponding to the loss of catechin units $\left(\mathrm{C}_{15} \mathrm{H}_{12} \mathrm{O}_{6}\right)$ [37]. According to previous reports [35,36], they were tentatively identified as procyanidin B1 (21), procyanidin B2 (24), fisetinidol- $(4 \alpha, 8)$-catechin (27) and procyanidin B3 (29) with the depronated ions at $m / z$ 305.06686, $m / z$ 577.13504, $m / z 577.13567, m / z 561.14044$ and $m / z$ 577.13543, respectively.

Miscellaneous compounds: Peak 15 was exactly identified as vanillin by comparison with a reference standard [33]. Peak 22 exhibited a deprotonated ion at $\mathrm{m} / z 181.05076$ corresponding to molecular formula of $\mathrm{C}_{9} \mathrm{H}_{10} \mathrm{O}_{4}$. The fragment ions at $m / z 163,135$ and 119 indicated the existence of hydroxyl, carbonyl and methoxyl groups. It was therefore identified as syringaldehyde [33]. Peak 37 displayed a $[\mathrm{M}-\mathrm{H}]^{-}$ion at $m / z 389.12421\left(\mathrm{C}_{20} \mathrm{H}_{22} \mathrm{O}_{8}\right)$, and the product ion of $\mathrm{m} / z 273 \mathrm{hinted}$ the presence of glucose group. It was tentatively identified as piceid [36]. Peak 40 with $\mathrm{C}_{15} \mathrm{H}_{12} \mathrm{O}_{2}$ gave fragments at $m / z 197$ and 185 in its MS spectrum, which were generated by losing $\mathrm{CH}_{2} \mathrm{O}$ and subsequently $\mathrm{H}_{2} \mathrm{O}$, and was identified as 3-methoxy-5-hydroxy-stilbene [36]. Peak 23 was $162 \mathrm{Da}$ more than peak 25 , and the product ions were very similar to peak 25 . Thus it was identified as 4-hydroxybenzoic acid-4-O- $\beta$-D-glucopyranoside [36].

\subsubsection{Comparison of Multiple Constituents}

UFLC/Q-TOF-MS has become a popular method to analyze constituents in complex systems due to the provided precise molecular weight and fragment characteristic information. Chemical profiles of $R$. roxburghii and $R$. sterilis fruits showed obviously differences based on the representative negative signals (Figure 3). The structures of identified compounds were shown in Figure 4.
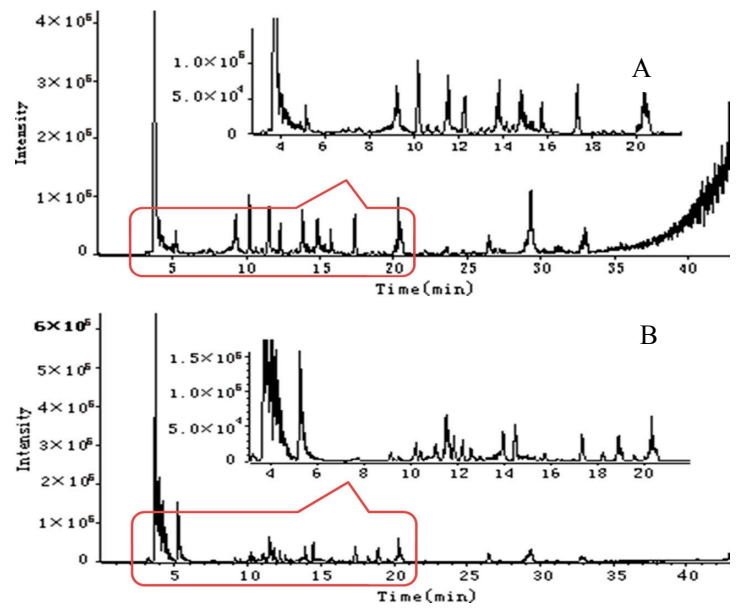

Figure 3. The representative total ion chromatograms of $R$. roxburghii (A) and R. sterilis (B) fruits obtained from UFLC/Q-TOF-MS in negative ion mode. 
<smiles>CC(O)C(=O)O</smiles>

Lactic acid (1)<smiles>O=C(O)CC(O)C(=O)O</smiles>

Malic acid (5)<smiles>O=C(O)c1ccc(O)c(O)c1</smiles>

Protocatechuic acid (9)<smiles>CCC(C)C(N)C(=O)O</smiles>

Isoleucine (13)<smiles>NC(CO)C(=O)O</smiles>

Serine (2)<smiles>O=C(O)C1(O)CC(O)C(O)C(O)C1</smiles>

Quinic acid (6)<smiles>O=C(O)CC(O)(CC(=O)O)C(=O)O</smiles>

Citric acid (10)<smiles>CC(C)CC(N)C(=O)O</smiles>

Leucine (14)<smiles>NC(Cc1ccccc1)C(=O)O</smiles>

Phenylalanine (17)<smiles>Oc1cc(O)c2c(c1)OC(c1cc(O)c(O)c(O)c1)C(O)C2</smiles>

Epigallocatechin (18)<smiles>N=C(N)NCCCC(N)C(=O)O</smiles>

Arginine (3)<smiles>CC(C)C(N)C(=O)O</smiles>

Valine (7)<smiles>NC(Cc1ccc(O)cc1)C(=O)O</smiles>

Tyrosine (11)<smiles>COc1cc(C=O)ccc1O</smiles>

Vanillin (15)<smiles>COc1cc(C(=O)O)cc(OC)c1O</smiles>

Syringic acid (19)<smiles>O=C(O)C1CCCN1</smiles>

Proline (4)<smiles>O=C1OC(C(O)CO)C(O)=C1O</smiles>

Ascorbic acid (8)<smiles>O=C(O)/C=C/c1ccc(O)cc1</smiles>

p-Coumaric acid (12)<smiles>O=C(O)c1cc(O)c(O)c(O)c1</smiles>

Gallic acid (16)<smiles>NC(Cc1c[nH]c2ccccc12)C(=O)O</smiles>

Tryptophan (20)<smiles>Oc1cc(O)c2c(c1)O[C@H](c1ccc(O)c(O)c1)C(O)C2c1c(O)cc(O)c2c1O[C@H](c1ccc(O)c(O)c1)[C@H](O)C2</smiles><smiles>COc1cc(C=O)cc(OC)c1O</smiles><smiles>[R]c1ccc(C(=O)O)cc1</smiles>

Procyanidin B1 (21)

Syringaldehyde (22) 4-Hydroxybenzoic acid-4-O- $\beta$-glucopyranoside (23): $\mathrm{R}=\mathrm{Oglc}$<smiles>Oc1cc(O)c2c(c1)O[C@H](c1ccc(O)c(O)c1)C(O)C2c1c(O)cc(O)c2c1O[C@H](c1ccc(O)c(O)c1)[C@H](O)C2</smiles>

Procyanidin B2 (24)<smiles>COc1cc(C(O)C(CO)Oc2c(OC)cc(C(=O)O)cc2OC)ccc1O</smiles>

Erythro-guaiacylglycerol $\beta$-sinapyl ether (26)<smiles>Oc1ccc2c(c1)O[C@H](c1ccc(O)c(O)c1)[C@H](O)[C@H]2c1c(O)cc(O)c2c1O[C@H](c1ccc(O)c(O)c1)C(O)C2</smiles>

Fisetinidol-( $4 \alpha, 8)$-catechin (27)

Figure 4. Cont. 
<smiles>O=C(O)/C=C/c1ccc(O)c(O)c1</smiles>

Caffeic acid (28)<smiles>Oc1cc(O)c2c(c1)OC(c1ccc(O)c(O)c1)C(O)C2c1c(O)cc(O)c2c1O[C@H](c1ccc(O)c(O)c1)C(O)C2</smiles>

Procyanidin B3 (29)<smiles>COc1cc(C2OCC3C(c4cc(OC)c(O)c(OC)c4)OCC23)cc(OC)c1O</smiles>

Diasyringaresinol (33)<smiles>[R2]c1cc(-c2oc3cc(O)cc(O)c3c(=O)c2[R])ccc1O</smiles>

\begin{tabular}{|c|c|c|}
\hline Compounds & $\mathbf{R}_{1}$ & $\mathbf{R}_{2}$ \\
\hline Rutin (30) & rutinose & $\mathrm{OH}$ \\
\hline Isoquercitin (31) & glucoside & $\mathrm{OH}$ \\
\hline Quercetin 3-O-[(6-O-3-hydroxy-3-methylglutaryl)- $\beta$-glucoside] (32) & (6-O-3-hydroxy-3-methylglutaryl)- $\beta$-glucoside & $\mathrm{OH}$ \\
\hline Quercetin-3-O-xyloside (34) & xyloside & $\mathrm{OH}$ \\
\hline Quercitrin (35) & rhamnose & $\mathrm{OH}$ \\
\hline Kaempferol 3-O-[(X-O-3-hydroxy-3-methylglutaryl)- $\beta$-galactoside] (36) & (X-O-3-hydroxy-3-methylglutaryl)- $\beta$-galactoside & $\mathrm{OH}$ \\
\hline Kaempferol 3-O-[(3-hydroxy-3-methylglutaryl)- $\beta$-glucuronide] (38) & (3-hydroxy-3-methylglutaroyl)- $\beta$-glucuronide & $\mathrm{H}$ \\
\hline Quercetin (44) & $\mathrm{OH}$ & $\mathrm{OH}$ \\
\hline Kaempferol (47) & $\mathrm{OH}$ & $\mathrm{H}$ \\
\hline Luteolin (49) & $\mathrm{H}$ & $\mathrm{H}$ \\
\hline
\end{tabular}<smiles>OCC1CC(Oc2cc(O)cc(C=Cc3ccc(O)cc3)c2)C(O)C(O)C1O</smiles>

Piceid (37)<smiles>O=C(CCc1ccc(O)cc1)C(Cc1cc(O)ccc1O)C1OC(CO)C(O)C(O)C1O</smiles>

Phlorizin (39)<smiles>COc1cc(O)cc(/C=C/c2ccccc2)c1</smiles>

3-Methoxy-5-hydroxy-stilbene (40)<smiles>COc1cc(C(O)C(CO)Oc2c(OC)cc(/C=C/CO)cc2OC)ccc1O</smiles>

Erythro/-guaiacylglycerol- $\beta$-O-4'-sinapyl ether (41)<smiles>O=C1CC(c2ccc(O)cc2)Oc2cc(O)cc(O)c21</smiles>

Dihydroapigenin (48)<smiles>CC/C=C/C/C=C/C/C=C/CCCCCCCC(=O)O</smiles>

9,12,15-Octadecatrienoic acid and (57)<smiles>COc1cc(C2OCC3C(c4ccc(O)c(OC)c4)OCC23)ccc1O</smiles>

Pinoresinol (42)<smiles>C=C(C)C1CCC2(C(=O)O)CCC3(C)C(=CCC4C5CCC6C(C)(CCC45C)CC(O)C(O)C6(C)C)C132</smiles>

$2 \alpha$,3 $\beta$-Dihydroxylup-20(29)-en-28-oic acid (56)<smiles>C#CCC=CCCCCCCCCC(=O)O</smiles>

9,12-Octadecadienoic acid (59)

Figure 4. Cont. 


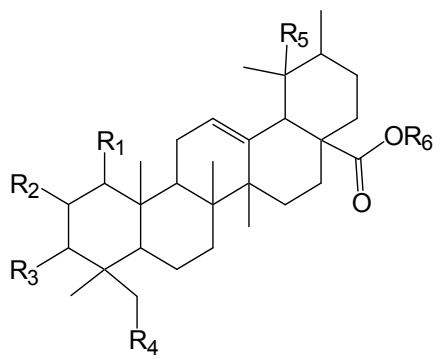

\begin{tabular}{|c|c|c|c|c|c|c|}
\hline Compounds & R1 & $\mathbf{R 2}$ & R3 & R4 & R5 & R6 \\
\hline \multicolumn{7}{|c|}{ Polygalacic acid 3-O- $\beta$-D-glucopyranoside (43) } \\
\hline \multicolumn{7}{|c|}{$19 \alpha$-Hydroxyasiatic acid-28-O- $\beta$-D-glucopyranoside (45) } \\
\hline Kajiichigoside F1 (46) & $\mathrm{H}$ & $\mathrm{OH}$ & $\mathrm{OH}$ & $\mathrm{H}$ & $\mathrm{OH}$ & glucose \\
\hline \multicolumn{7}{|c|}{ 1-Hydroxyeuscaphic acid (50) } \\
\hline \multicolumn{7}{|c|}{$2 \alpha, 19 \alpha$-Dihydroxy-3-oxo-urs-12-en-28-oic acid (51/53) } \\
\hline Euscaphic acid (52) & $\mathrm{H}$ & $\mathrm{OH}$ & $\mathrm{OH}$ & $\mathrm{OH}$ & $\mathrm{H}$ & $\mathrm{H}$ \\
\hline Pomolic acid (54/55) & $\mathrm{H}$ & $\mathrm{H}$ & $\mathrm{OH}$ & $\mathrm{H}$ & $\mathrm{OH}$ & $\mathrm{H}$ \\
\hline Ursolic acid (58) & $\mathrm{H}$ & $\mathrm{H}$ & $\mathrm{OH}$ & $\mathrm{H}$ & $\mathrm{H}$ & $\mathrm{H}$ \\
\hline
\end{tabular}

Figure 4. Chemical structures of compounds identified in the methanol extracts of $R$. roxburghii and R. sterilis fruits.

Organic acids were the main chemotypes reported in R. roxburghii fruit, especially its high ascorbic acid level. Huang et al. demonstrated that the content of ascorbic acid in $R$. roxburghii fruit is higher than that in most common fruit crops, e.g., tomato $(\sim 20 \mathrm{mg})$, strawberry $(\sim 50 \mathrm{mg})$, and kiwifruit ( $\sim 100 \mathrm{mg}$ ) [38]. In this study, a high content of ascorbic acid was also found and determined by a LC-MS method [39]. Comparing the difference of organic acid compositions between $R$. roxburghii and $R$. sterilis fruits, compound 57, namely 9,12,15-octadecatrienoic acid was only detectable in $R$. sterilis fruit, which was consistent with the result of GC-MS; whereas, syringic acid (19) was only found in $R$. roxburghii fruit rather than in $R$. sterilis fruit.

Flavonoids were the second main constituents in both $R$. roxburghii and $R$. sterilis fruits. The radioprotective effects of flavonoids from $R$. roxburghii fruit has been proved by cell model and animal experiments [5]. There were 11 flavonoids, including quercetin, kaempferol and their derivatives found in R. roxburghii and R. sterilis fruits. However, epigallocatechin (18) was only found in $R$. sterilis fruit rather than in $R$. roxburghii fruit.

More than twenty triterpenes have so far been previously found in the Rose family [34]. Triterpenes were other main constituents in $R$. roxburghii fruit with $\alpha$-glucosidase inhibitory activity [40]. Liang et al. [14] reported that four kinds of triterpenes were isolated from $R$. sterilis fruit three decades ago. In this study, eleven triterpenes were shared between $R$. roxburghii and $R$. sterilis fruits. In addition, polygalacic acid 3-O- $\beta$-D-glucopyranoside (43), 19a-hydroxyasiatic acid-28-O- $\beta$-D-glucopyranoside (45), $2 \alpha, 19 \alpha$-dihydroxy-3-oxo-urs-12-en-28-oic acid (53), 2 $\alpha, 3 \beta$-dihydroxylup-20(29)-en-28-oic acid (56) and ursolic acid (58) were reported for the first time in $R$. sterilis fruit.

Amino acids as nutrients have various functions in human beings. In addition, the amino acid composition plays an important role affecting the flavor of fruits. In term of the types of compounds, there was no difference between $R$. roxburghii and $R$. sterilis fruits [18].

A total of five phenylpropanoid derivatives were identified in $R$. roxburghii and $R$. sterilis fruits. Among them, diasyringaresinol (33) and phlorizin (39) were only found in $R$. roxburghii fruit.

Tannins are a kind of secondary metabolite common reported in the genus Rosa, and most of them are characterized by catechin and epicatechin as constitutive units. For example, Yan et al. [36] reported five condensed tannins in $R$. laevigata, namely procyanidin B3, fisetinidol- $(4 \alpha, 8)$-catechin,

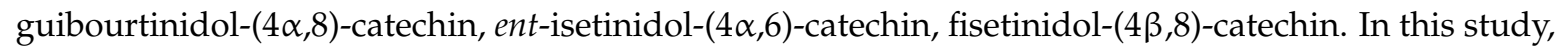
procyanidin B1 (21), procyanidin B2 (24), and procyanidin B3 (29) were commonly found in $R$. roxburghii and R. sterilis fruits. Interestingly, fisetinidol-( $4 \alpha, 8)$-catechin (27) was only found in $R$. sterilis fruit. 
Five miscellaneous compounds, including two benzaldehyde derivatives (15 and 22), two stilbenes (37 and 40) and one benzoic acid derivative (23), were detected in this study. Except for the two stilbenes, the presence of the three compounds in $R$. roxburghii and $R$. sterilis fruits was different although they have been found in Rosa species before. Vanillin (15) and syringaldehyde (22) were only detected in $R$. roxburghii fruit and 4-hydroxybenzoic acid-4-O- $\beta$-D-glucopyranoside (23) only existed in $R$. sterilis fruit.

Generally, 50 phytochemicals existed in both $R$. roxburghii and $R$. sterilis fruits. Five characteristic compounds, including vanillin (15), syringic acid (19), syringaldehyde (22), diasyringaresinol (33) and phlorizin (39) were found in $R$. roxburghii fruit and the other four compounds, including epigallocatechin (18), 4-hydroxybenzoic acid-4-O-glucopyranoside (23), fisetinidol-(4 $\alpha, 8)$-catechin (27), and 9,12,15-octadecatrienoic acid (57) were only detectable in $R$. sterilis fruit. The nine characteristic phytochemicals were considered to be potential markers for discriminating $R$. roxburghii fruit from $R$. sterilis fruit in quality control. It is general known that phytochemicals are responsible for the bioactivities of medicinal plants. Clarifying the characteristic phytochemicals in $R$. roxburghii and $R$. sterilis fruits is very important for their development and application in health-related industries.

\section{Materials and Methods}

\subsection{Chemicals and Reagents}

Methanol (HPLC grade) and n-hexane (GC grade) were purchased from Merck KGaA (Darmstadt, Germany). Distilled water was from Guangzhou Watson's Food \& Drinks Co., Ltd. (Guangzhou, China). The other reagents used were of analytical grade and were used without any further purification. Gallic acid, 4-hydroxybenzoic acid, caffeic acid, protocatechuic acid, isoquercetin, quercitrin, quercetin and kaempferol were purchased from the National Institute for the Control of Pharmaceutical and Biological Products (Beijing, China). Lactic acid, malic acid, ascorbic acid, citric acid, tryptophan, rutin and luteolin were purchased from Aladdin Industrial Corporation (Shanghai, China). Serine, arginine, proline, valine, tyrosine, leucine, isoleucine, phenylalanine, p-coumaric acid, vanillin and syringic acid were purchased from Sigma-Aldrich Chemical Co. (St. Louis, MO, USA). n-Hexadecanoic acid, 9,12,15-octadecatrienoic acid, 9,12-octadecadienoic acid, and 9-octadecenoic acid were purchased from Dr. Ehrenstorfer GmbH (Augsburg, Germany). The purities of all compounds were over $95 \%$, as determined by the area normalization method using HPLC or GC.

\subsection{Plant Material}

$R$. roxburghii and $R$. sterilis were cultivated in Guizhou province and their fruits for each species were collected from five cultivars in July (R. roxburghii) and October (R. sterilis), 2015, respectively. All of the specimens were exactly identified by the authors and stored in $-80{ }^{\circ} \mathrm{C}$ in Guangzhou Institute of Advanced Technology, Chinese Academy of Sciences, China.

\subsection{GC-MS Experiment}

\subsubsection{Sample Preparation}

The fresh fruit was washed three times with distilled water and cut randomly into small pieces. Then $100 \mathrm{~g}$ of the chipped sample (20 $\mathrm{g}$ from each cultivar) was stirred in a bullet blender (Jiuyang Co., Ltd., Shandong, China) for $1 \mathrm{~min}$. After adding $200 \mathrm{~mL}$ distilled water, the sample was subjected to hydrodistillation in a steam distillation vessel for $3 \mathrm{~h}$. The oil was extracted by $2 \mathrm{~mL}$ hexane and dried with excess anhydrous sodium sulphate. Finally, the oil extract was filtered by $0.22 \mu \mathrm{m}$ microporous membrane and stored at $4{ }^{\circ} \mathrm{C}$ before injection [41]. 


\subsubsection{Instrument Conditions}

The analysis was carried out with an 7890A-5975C GC-MSD instrument (Agilent, Santa Clara, CA, USA). Separations were performed using an Agilent HP-5MS capillary column $(30 \mathrm{~m} \times 250 \mathrm{~mm} \times 0.25 \mu \mathrm{m})$. The $\mathrm{GC}$ oven temperature was programmed from $50{ }^{\circ} \mathrm{C}(1 \mathrm{~min}$ isothermal) to $160{ }^{\circ} \mathrm{C}$ at $15^{\circ} \mathrm{C} \cdot \mathrm{min}^{-1}$, and then to $280{ }^{\circ} \mathrm{C}$ at $5^{\circ} \mathrm{C} \cdot \mathrm{min}^{-1}(5 \mathrm{~min}$ isothermal). A $5 \mu \mathrm{L}$ sample solution was injected into GC system without split. Helium was the carrier gas with $2 \mathrm{~mL} / \mathrm{min}$. The injector temperature was set to $280{ }^{\circ} \mathrm{C}$. The MS was operated in full scan mode (50-500 amu at $0.5 \mathrm{scan} / \mathrm{s}$ ) with electron ionization mode at $70 \mathrm{eV}$.

\subsubsection{Data Analysis}

Data were evaluated by MSD ChemStation E.02.02.1431 software (Agilent). The identification of the compounds was carried out by comparing with data known from the literature, the NIST 05 library (NIST Mass Spectral Database, PC-Version 5.0, 2005, National Institute of Standards and Technology, Gaithersburg, MD, USA) and authentic standards. Percentage data of the total ion current chromatograms were calculated by the area normalization method without applying response factor correction and shown as mean \pm standard deviation (SD).

\subsection{UFLC/Q-TOF-MS Experiments}

\subsubsection{Sample Preparation}

Three hundred gram of fresh fruits ( $60 \mathrm{~g}$ from each cultivar) were washed three times with distilled water and cut into small pieces (thickness about $0.1 \mathrm{~cm}$ ) and then lyophilized by Modulyo vacuum freeze-drying (Thermo Fisher Scientific Inc., Waltham, MA, USA). During the whole freeze-drying process, the temperature was kept at $-50{ }^{\circ} \mathrm{C}$ with vacuum degree of $10 \mathrm{mbar}$ for $48 \mathrm{~h}$. Then the dried samples were crushed by Media BM252C blender (Midea Group Ltd., Guangdong, China) and stored at $-20^{\circ} \mathrm{C}$ until use. One gram of the dried fine powder was accurately weighed and extracted with $25 \mathrm{~mL}$ methanol twice in a KQ600DE ultrasonic bath (Kunshan, Jiangsu, China) for $30 \mathrm{~min}$ at room temperature. After filtered, the combined extract was dilute with methanol to $50 \mathrm{~mL}$ and stored at $4{ }^{\circ} \mathrm{C}$ for further study.

\subsubsection{Instrument Conditions}

The multiple constituents in the methanol extraction of $R$. roxburghii and $R$. sterilis fruits were identified by UFLC/Q-TOF-MS method. Chromatographic analysis was performed on a UFLC XR system (Shimadzu Corporation, Kyoto, Japan) equipped with a LC-20AD-XR binary pump, SIL-20AD-XR autosampler and a CTO-20A column oven. The column was an Agilent Eclipse Plus $\mathrm{C} 18\left(2.1 \mathrm{~mm} \times 100 \mathrm{~mm}, 1.8 \mu \mathrm{m}\right.$, Agilent), maintained at $35^{\circ} \mathrm{C}$. The sample was eluted at a flow rate of $0.2 \mathrm{~mL} / \mathrm{min}$ in a linear gradient mode of $\mathrm{A}(0.1 \%$ formic acid:water $)$ and $\mathrm{B}(0.1 \%$ formic acid:methanol): $0-40 \mathrm{~min}(5 \%-100 \% \mathrm{~B})$, then kept for $3 \mathrm{~min}$ at $100 \%$ B. The injection volume was $5 \mu \mathrm{L}$. Mass spectrometry was performed on the triple TOF 5600 (AB SCIEX, Foster City, CA, USA) a hybrid triple quadrupole time-of-flight mass spectrometer equipped with ESI source, and mass range was set at $m / z$ 100-1200. The experiment parameters were as follows: CUR: 35; GS1: 55; GS21 55; ISVF: 4500; TEM: 550. Nitrogen was used as nebulizer and auxiliary gas. All the acquisition and analysis of data were controlled by the Peak View Software TM V.1.6 (AB SCIEX).

\subsubsection{Data Analysis}

Compound identification of methanol extracts of R. roxburghii and R. sterilis fruits was carried out by comparison of MS and collision-induced dissociation (CID) spectral data of analytes with those of reference standards. When standard substances were unavailable, compounds were tentatively identified by precise molecular weight within an accuracy of 5 ppm or less which can be uniquely 
associated with a specific molecular formula. At the same time, based on the characteristic pyrolysis fragments obtained by CID spectra, compound structures were assigned by comparison with literature data and standards with similar structures.

\section{Conclusions}

This study analyzed and compared the important dietary constituents in $R$. roxburghii and $R$. sterilis fruits, including essential oils, organic acids, flavonoids, triterpenes, amino acids, phenylpropanoid derivatives, condensed tannins, stilbenes, benzaldehyde derivatives and a benzoic acid derivative. The phytochemical profiles and the chemical differences of these two fruits have been generally illustrated. However, a series of $R$. roxburghii and $R$. sterilis fruits, as the various samples, should be collected from the different producing areas for further study. The contents of main constituents and characteristic compounds, along with their bioactivities, need further in-depth study.

Acknowledgments: The study was supported by the Open Project of Guangdong Provincial Key Laboratory of New Drug Screening, the PhD Start-up Fund of Natural Science Foundation of Guangdong Province (2015A030310139) and the Science and Technology Program of Guangzhou (2014J4100045).

Author Contributions: Jing-yu He and Wei-ming Fu conceived and designed the experiments; Meng-hua Liu, Qi Zhang, Yuan-he Zhang and Jing-yu He performed the experiments; Meng-hua Liu and Qi Zhang analyzed the data; Meng-hua Liu and Xian-yuan Lu wrote the manuscript; Jing-yu He and Wei-ming Fu modified the manuscript.

Conflicts of Interest: The authors declare no conflict of interest.

\section{References}

1. Yang, H.; Hu, J.W.; Huang, X.F.; Zhou, C.; Li, L.Y.; Fan, M.Y. Risk assessment of heavy metals pollution for Rosa sterilis and soil from planting bases located in karst areas of Guizhou province. Appl. Mech. Mater. 2015, 700, 475-481. [CrossRef]

2. Westhuizen, F.H.V.D.; Rensburg, C.S.J.V.; Rautenbach, G.S.; Marnewick, J.L.; Du, T.L.; Huysamen, C.; Louw, R.; Pretorius, P.J.; Erasmus, E. In vitro antioxidant, antimutagenic and genoprotective activity of Rosa roxburghii fruit extract. Phytother. Res. 2008, 22, 376-383. [CrossRef] [PubMed]

3. Zhang, C.; Liu, X.; Qiang, H.; Ke, L.; Wang, J.; Chen, D.; Zhang, Y. Inhibitory effects of Rosa roxburghii tratt juice on in vitro oxidative modification of low density lipoprotein and on the macrophage growth and cellular cholesteryl ester accumulation induced by oxidized low density lipoprotein. Clin. Chim. Acta 2001, 313, 37-43. [CrossRef]

4. Chen, Y.; Liu, Z.J.; Liu, J.; Liu, L.K.; Zhang, E.S.; Li, W.L. Inhibition of metastasis and invasion of ovarian cancer cells by crude polysaccharides from Rosa roxburghii tratt in vitro. Asian Pac. J. Cancer Prev. 2014, 15, 10351-10354. [CrossRef] [PubMed]

5. Xu, P.; Zhang, W.B.; Cai, X.H.; Lu, D.D.; He, X.Y.; Qiu, P.Y.; Wu, J. Flavonoids of Rosa roxburghii tratt act as radioprotectors. Asian Pac. J. Cancer Prev. 2014, 15, 8171-8175. [CrossRef] [PubMed]

6. Janse van Rensburg, C.; Erasmus, E.; Loots, D.T.; Oosthuizen, W.; Jerling, J.C.; Kruger, H.S.; Louw, R.; Brits, M.; van der Westhuizen, F.H. Rosa roxburghii supplementation in a controlled feeding study increases plasma antioxidant capacity and glutathione redox state. Eur. J. Nutr. 2005, 44, 452-457. [CrossRef] [PubMed]

7. Li, Q.; Nan, Y.; Qin, J.; Yang, Y.; Hao, X.; Yang, X. Chemical constituents from medical and edible plants of Rosa roxburghii. China J. Chin. Mater. Med. 2016, 41, 451-455.

8. Chen, Q.; Gao, J. Analysis of volatile components from Rosa roxburghii tratt seed by HS-SPME and GC-MS. China Brew. 2014, 33, 141-142.

9. Sarangowa, O.; Kanazawa, T.; Nishizawa, M.; Myoda, T.; Bai, C.; Yamagishi, T. Flavonol glycosides in the petal of Rosa species as chemotaxonomic markers. Phytochemistry 2014, 107, 61-68. [CrossRef] [PubMed]

10. Dai, T.; Li, Q.J.; Nan, Y.; Yang, X.S. Chemical Components of Antioxidant Activity Parts of Rosa roxburghii Fruit. Chin. J. Exp. Tradit. Med. Formulae 2015, 11, 62-65.

11. Luo, D.Y. Exploration and Research of Cili; People's Publishing House in Guizhou: Guiyang, China, 1987; Volume 12, p. 66. 
12. An, H.M.; Liu, M.; Yang, M.; Fan, W.G. Analysis of main organic acid compositions in Rosa roxburghii Tratt. Sci. Agric. Sin. 2011, 44, 2049-2100.

13. Qiang, X.; Wen, X.; Tao, N.; Hu, Z.; Yue, H.; Deng, X. Extraction of high quality of RNA and construction of a suppression subtractive hybridization (SSH) library from chestnut rose (Rosa roxburghii tratt). Biotechnol. Lett. 2006, 28, 587-591.

14. Liang, G.Y.; Gray, A.I.; Waterman, P.G. Pentacyclic triterpenes from the fruits of Rosa sterilis. J. Nat. Prod. 1989, 52, 162-166.

15. Wen, X.P.; Deng, X.Y. Characterization of genotypes and genetic relationship of Cili (Rosa roxburghii) and its relatives using RAPD markers. J. Agric. Biotechnol. 2003, 22, 376-383.

16. Zhang, D.; Wei, G.X.; Wang, W.; Feng, F.; Zeng, F.K. Comparative research on basic ingredients and volatile aroma compounds of Rosa roxburghii Tratt and Rosa sterilis D. shi. Sci. Technol. Food Ind. 2016. [CrossRef]

17. Fu, H.X.; Wang, D.P.; Huang, L.R.; Ma, L.; Yang, X.S. Analysis of the Volatile Aroma Compounds of Rosa Roxburghii Tratt and Rosa sterilis. Fine Chem. 2012, 29, 875-878.

18. Lu, M.; An, H.; Zhao, X. Analysis of Amino Acids in Rosa sterilis and Rosa roxburghii Fruits. Food Sci. 2015, 36, 118-121.

19. Klavina, L.; Springe, G.; Nikolajeva, V.; Martsinkevich, I.; Nakurte, I.; Dzabijeva, D.; Steinberga, I. Chemical composition analysis, antimicrobial activity and cytotoxicity screening of Moss extracts (Moss Phytochemistry). Molecules 2015, 20, 17221-17243. [CrossRef] [PubMed]

20. Liu, M.; Li, P.; Zeng, X.; Wu, H.; Su, W.; He, J. Identification and pharmacokinetics of multiple potential bioactive constituents after oral administration of Radix Astragali on cyclophosphamide-induced immunosuppression in Balb/c mice. Int. J. Mol. Sci. 2015, 16, 5047-507. [CrossRef] [PubMed]

21. Ye, J.H.; Liu, M.H.; Zhang, X.L.; He, J.Y. Chemical profiles and protective effect of Hedyotis diffusa Willd in lipopolysaccharide-induced renal inflammation mice. Int. J. Mol. Sci. 2015, 16, 27252-27269. [CrossRef] [PubMed]

22. Liu, M.H.; Ko, C.H.; Ma, N.; Tan, P.W.; Fu, W.M.; He, J.Y. Chemical profiles, antioxidant and anti-obesity effects of extract of Bambusa textilis Mcclure leaves. J. Funct. Foods 2016, 22, 533-546. [CrossRef]

23. Liang, L.L.; Han, L.; Chen, X.; Shi, L.H. Study on volatile Components in Rose roxburghii fruit. Chem. Bull. 1992, 5, 34-36.

24. Jiang, Y.H.; Gao, J.; Zhao, P.; Zhang, D.G.; Li, Y.H. GC-MS Analysis of Volatile Components from the Fresh Fruits of Rosa sterilis. Food Res. Dev. 2013, 34, 91-94.

25. Wu, X.Q.; Lu, H.; Jin, J.L.; Xie, P.; Wang, S.S.; Yang, S.J. GC-MS Analysis of Volatile Oil from Rosa sterilis by Supercritical $\mathrm{CO}_{2}$ Extraction. Chin. J. Exp. Tradit. Med. Formulae 2014, 20, 98-101.

26. Michelini, F.M.; Ramírez, J.A.; Berra, A.; Galagovsky, L.R.; Alché, L.E. Anti-herpetic and anti-inflammatory activities of two new synthetic 22,23-dihydroxylated stigmastane derivatives. J. Steroid Biochem. Mol. Biol. 2008, 111, 111-116. [CrossRef] [PubMed]

27. Bourourou, M.; Heurteaux, C.; Blondeau, N. Alpha-linolenic acid given as enteral or parenteral nutritional intervention against sensorimotor and cognitive deficits in a mouse model of ischemic stroke. Neuropharmacology 2016, 108, 60-72. [CrossRef] [PubMed]

28. Kim, M.; Kim, M.; Lee, Y.J.; Lee, S.P.; Kim, T.S.; Yang, H.J.; Kwon, D.Y.; Lee, S.H.; Lee, J.H. Effects of $\alpha$-linolenic acid supplementation in perilla oil on collagen-epinephrine closure time, activated partial thromboplastin time and lp-pla2 activity in nondiabetic and hypercholesterolemic subjects. J. Funct. Foods 2016, 23, 95-104. [CrossRef]

29. Domenichiello, A.F.; Kitson, A.P.; Chen, C.T.; Trépanier, M.O.; Stavro, P.M.; Bazinet, R.P. The effect of linoleic acid on the whole body synthesis rates of polyunsaturated fatty acids from $\alpha$-linolenic acid and linoleic acid in free-living rats. J. Nutr. Biochem. 2016, 30, 167-176. [CrossRef] [PubMed]

30. Cunja, V.; Mikulic-Petkovsek, M.; Zupan, A.; Stampar, F.; Schmitzer, V. Frost decreases content of sugars, ascorbic acid and some quercetin glycosides but stimulates selected carotenes in Rosa canina hips. J. Plant Physiol. 2015, 178, 55-63. [CrossRef] [PubMed]

31. Nađpal, J.D.; Lesjak, M.M.; Šibul, F.S.; Anačkov, G.T.; Četojević-Simin, D.D.; Mimica-Dukić, N.M.; Beara, I.N. Comparative study of biological activities and phytochemical composition of two rose hips and their preserves: Rosa canina L. and Rosa arvensis huds. Food Chem. 2016, 192, 907-914. [CrossRef] [PubMed] 
32. Porter, E.A.; Bos, A.A.V.D.; Kite, G.C.; Veitch, N.C.; Simmonds, M.S.J. Flavonol glycosides acylated with 3-hydroxy-3-methylglutaric acid as systematic characters in Rosa. Phytochemistry 2012, 81, 90-96. [CrossRef] [PubMed]

33. Li, X.; Cao, W.; Shen, Y.; Li, N.; Dong, X.P.; Wang, K.J.; Cheng, Y.X. Antioxidant compounds from Rosa laevigata fruits. Food Chem. 2012, 130, 575-580. [CrossRef]

34. Gao, P.Y.; Li, L.Z.; Peng, Y.; Piao, S.J.; Zeng, N.; Lin, H.W.; Song, S.J. Triterpenes from fruits of Rosa laevigata. Biochem. Syst. Ecol. 2010, 38, 457-459. [CrossRef]

35. Yan, M.; Zhu, Y.; Zhang, H.J.; Jiao, W.H.; Han, B.N.; Liu, Z.X.; Qiu, F.; Chen, W.S.; Lin, H.W. Anti-inflammatory secondary metabolites from the leaves of Rosa laevigata. Bioorg. Med. Chem. 2013, 21, 3290-3297. [CrossRef] [PubMed]

36. Yan, G.; Li, S.; Hu, J.; Zhai, X.; Ma, W.; Li, N.; Wang, K. Phenolic constituents from the roots of Rosa laevigata (Rosaceae). Biochem. Syst. Ecol. 2014, 52, 23-26. [CrossRef]

37. Riffault, L.; Destandau, E.; Pasquier, L.; André, P.; Elfakir, C. Phytochemical analysis of Rosa hybrida, cv. 'jardin de granville' by HPTLC, HPLC-DAD and HPLC-ESI-HRMS: polyphenolic fingerprints of six plant organs. Phytochemistry 2014, 99, 127-134. [CrossRef] [PubMed]

38. Huang, M.; Xu, Q.; Deng, X.X. L-ascorbic acid metabolism during fruit development in an ascorbate-rich fruit crop chestnut rose (Rosa roxburghii tratt). J. Plant Physiol. 2014, 171, 1205-1216. [CrossRef] [PubMed]

39. He, J.Y.; Zhang, Y.H.; Ma, N.; Zhang, X.L.; Liu, M.H.; Fu, W.M. Comparative analysis of multiple ingredients in Rosa roxburghii and $R$. sterilis fruits and their antioxidant activities. J. Funct. Foods 2016. [CrossRef]

40. Qin, J.J.; Li, Q.J.; Xue, Y.; Ma, L.; Yang, X.S. Study on extraction methods and $\alpha$-glucosidase inhibitory activity from the total triterpenes of Rosa roxbughii. Sci. Technol. Food Ind. 2014, 10, 186-189.

41. Han, L.; Chen, X. Study on extraction methods for volatile flavor compounds in fresh fruit of Rosa roxburghii Tratt. Sci. Technol. Food Ind. 2007, 28, 100-101.

Sample Availability: Samples of the compounds are available from the authors.

(C) 2016 by the authors; licensee MDPI, Basel, Switzerland. This article is an open access article distributed under the terms and conditions of the Creative Commons Attribution (CC-BY) license (http://creativecommons.org/licenses/by/4.0/). 